1

11 Keywords: Toxoplasma gondii, Apicomplexa, flippase, P4-ATPase, cell division control

12 protein 50, microneme exocytosis, invasion, egress, phosphatidylserine.

13

14 Running title: Contribution of phosphatidylserine in microneme exocytosis

15

16

17 of Geneva, 1 Rue Michel-Servet, 1206 Geneva, Switzerland

19

20

a Present address: Information Génomique \& Structurale, CNRS, Aix-Marseille Université,

21 Marseille, France.

22

23

24

\section{critically participates in microneme exocytosis \\ Toxoplasma gondii phosphatidylserine flippase complex ATP2B-CDC50.4} Hugo Bisio $^{1 a}$, Aarti Krishnan ${ }^{1}$, Jean-Baptiste Marq ${ }^{1} \&$ Dominique Soldati-Favre ${ }^{1 *}$

\section{Affiliation}

${ }^{1}$ Department of Microbiology and Molecular Medicine, CMU, Faculty of Medicine, University

*Corresponding author E-mail: dominique.soldati-favre@unige.ch 


\section{Summary}

26 Regulated microneme secretion governs motility, host cell invasion and egress in the obligate

27 intracellular apicomplexans. Intracellular calcium oscillations and phospholipid dynamics

28 critically regulate micronemes exocytosis. Despite its importance for the lytic cycle of these

29 parasites, molecular mechanistic details about exocytosis are still missing. Some members of

30 the P4-ATPases act as flippases, changing the phospholipid distribution by translocation from

31 the outer to the inner leaflet of the membrane. Here, the localization and function of the

32 repertoire of P4-ATPases was investigated across the lytic cycle of Toxoplasma gondii. Of

33 relevance, ATP2B and the non-catalytic subunit cell division control protein 50.4 (CDC50.4)

34 form a stable heterocomplex at the parasite plasma membrane, essential for microneme

35 exocytosis. This complex is responsible for flipping phosphatidylserine (PS), which presumably

36 acts as a lipid mediator for the organelle fusion with the plasma membrane. DOC2.1, a

37 previously described key egress and invasion factor, is shown here to be affected in its function

38 in egress upon mutation on residues putatively involved in calcium binding. This study points

39 toward the importance of PS in microneme exocytosis and unveils subtle differences in the

40 signaling cascades leading to organelle secretion between intracellular and extracellular

41 parasites to ensure egress and invasion, respectively. 


\section{Author Summary}

54 Biological membranes display diverse functions, including membrane fusion, which are

55 conferred by a defined composition and organization of proteins and lipids. Apicomplexan

56 parasites possess specialized secretory organelles (micronemes), implicated in motility,

57 invasion and egress from host cells. Microneme exocytosis is already known to depends on

58 phosphatidic acid for its fusion with the plasma membrane. Here we identify a type P4-ATPase

59 and its CDC50 chaperone (ATP2B-CDC50.4) that act as flippase and contribute to the

60 enrichment of phosphatidylserine (PS) in the inner leaflet of the parasite plasma membrane. PS

61 and the previously described C2-containing protein DOC2.1 differentially participate in

62 microneme exocytosis in the context of environmental changes. Overall, our results shed light

63 on the importance membrane homeostasis and lipid composition in controlling microneme

64 secretion. 


\section{Introduction}

The phylum of Apicomplexa encompasses a diverse group of obligate intracellular protozoan parasites responsible for severe diseases in animals and humans. Plasmodium spp., the etiological agent of malaria, account for half a million deaths per year (World Malaria Report 2017, WHO). Cryptosporidium is one of the most important agents causing severe diarrhea in children (1). Relevant for the farming industry, Eimeria and Theileria are responsible for a considerable economic burden $(2,3)$. Toxoplasma gondii is the most ubiquitous member of the phylum, capable of infecting humans and animals. The lytic cycle of apicomplexan parasites is tightly controlled to ensure parasite survival and dissemination $(4,5)$. Underpinning several steps of the lytic cycle is the release of apical secretory organelles called micronemes that are conserved in all motile and invasive stages of apicomplexans (4). The micronemes secrete adhesins, perforins and proteases that allow gliding, invasion and egress of the parasites $(6,7)$. In a simplified model, microneme exocytosis is regulated and initiated by the production of cyclic guanosine monophosphate (cGMP) via a signaling platform composed of an atypical guanylate cyclase (GC) fused to a P type-IV ATPase (P4-ATPase) and associated to CDC50.1 as well as to a unique GC organizer, UGO (8-11). The level of cGMP is tightly controlled by phosphodiesterases, which differential phosphorylation state upon depletion of the protein cAMP-dependent protein kinase (PKA-C1) indicates that are presumably regulated by cyclic adenosine monophosphate (cAMP) levels $(12,13)$. In turn, cGMP leads to the activation of the cGMP-dependent protein kinase (PKG) (14) which triggers a signaling cascade that involves the production of inositol-tri-phosphate (IP3) and diacylglycerol (DAG) by phosphoinositide phospholipase C (PI-PLC) (15). IP3 is believed to mobilize calcium from an unknown intracellular store of the parasite (16) and activate calcium-dependent protein kinases (CDPKs) $(17,18)$. Both CDPK1 and CDPK3 contribute to microneme exocytosis while CDPK1 additionally extrudes the conoid and activates the actomyosin system (17-19), allowing parasite 
90 gliding motility, invasion and egress (20). On the other hand, DAG is converted into

91 phosphatidic acid (PA) through a reversible reaction catalyzed by the DAG kinase 1 (DGK1)

92 and PA phosphatases (PAPs) (21). Importantly, several feedback loops are expected to feed into

93 different steps of this signaling pathway.

94 The endomembrane system of the cell displays diverse functions conferred by a defined 95 composition and organization of proteins and lipids. In particular, the exocytosis of secretory 96 organelles depends on specific phospholipids that select the target membranes and trigger 97 fusion. In apicomplexan parasites, PA acts as an essential lipid mediator for microneme exocytosis $(21,22)$. PA is produced in the inner leaflet of the plasma membrane and allows the docking of micronemes with the assistance of the acylated pleckstrin-homology domaincontaining protein $(\mathrm{APH})$ on the microneme organelle surface $(21,23)$. Importantly, the asymmetric distribution of phospholipids (PLs) across the plasma membrane is known to generate a physical surface tension that is used to induce membrane curvature, favoring vesicle budding and fusion (24). These gradients are set and maintained by different groups of proteins including P4-ATPases, which function as flippases and form heterodimeric complexes with cell division control protein 50 (CDC50), that act as cofactors and chaperones (Figure 1A) (25). P4ATPases possess ten predicted transmembrane spanning domains with cytosolic domains mediating nucleotide binding $(\mathrm{N})$, phosphorylation $(\mathrm{P})$ and dephosphorylation (A) (Figure 1A) (26). The P4-ATPases couple the hydrolysis of ATP with inward PLs translocation via a PostAlbers mechanism (25), transitioning between two intermediate states: E1 and E2, with different affinities to substrates. During this process, the transmembrane region

111 remainsstructurally rigid based on its interaction with the CDC50 partner (27). Among the 112 repertoire of P4-ATPases in apicomplexan parasites, some are essential for parasite survival $113(28-30)$ yet little is known about their biological roles and enzymatic functions. Beside the lipid 114 composition, membrane fusion is a universal process that also involves a machinery composed 
of SNAREs (for "soluble N-Ethylmaleimide-sensitive factor (NSF)-attachment protein

116 receptor") (31). During fusion, vesicular and target SNAREs assemble into an $\alpha$-helical trans-

117 SNARE complex that forces the two membranes tightly together (31). Additionally, this machinery is controlled by C2-containing proteins, like synaptotagmin/ferlin and DOC2, in a calcium-dependent manner $(32,33)$. The SNARE proteins appear to play pleiotropic functions in $T$. gondii with none identified to date to be uniquely associated to microneme secretion (34). Contrastingly, both DOC2.1 and Ferlin 1 (FER1) are solely dedicated to microneme secretion in Plasmodium spp. and T. gondii (35-38). While DOC2.1 function strictly participates in microneme exocytosis (35), FER1 is involved in microneme proteins trafficking in addition to exocytosis (37), although some of these phenotypes might result from indirect dominant negative effects.

In this study, we address the importance of several P type-IV ATPases and CDC50 chaperones in T. gondii. We show that ATP2B forms a heterocomplex with CDC50.4 and acts as an essential flippase to maintain phosphatidylserine (PS) enrichment in the inner leaflet of the parasite plasma membrane. ATP2B and CDC50.4 crucially contribute to microneme exocytosis indicating that PS is a key lipid participating in microneme fusion with the plasma membrane. In this context, DOC2.1, which due to its $\mathrm{C} 2$ domain could bind phospholipids, primes the micronemes for secretion only in intracellular parasites triggered to egress through its putative calcium binding residues.

\section{Results}

136 Identification and localization of the putative flippases and their CDC50 partners in

\section{Toxoplasma gondii}

138 Sequence homology search in T. gondii genome identified six genes predicted to code for P4139 ATPases and four genes for CDC50 cofactors (Figure 1B and Table S1). We utilized the 
nomenclature already established for Plasmodium species in order to name the putative homologues of P4-ATPases (39). We have chosen this nomenclature, instead of the one presented in a recent overlapping report (30), in order to provide an integrative view of different apicomplexan parasites P4-ATPases and facilitate comparisons. ATP2A and ATP2B appear to

144 be paralogues that are present either as one or two copies across all members of the Apicomplexa phylum. Similarly, ATP7A and ATP7B are putative paralogues found as a pair in the cyst-forming coccidian subgroup of Apicomplexa but absent in Theileria and Cryptosporidia. Plausible orthologs of T. gondii ATP8 and GC are found across the phylum (Figure 1B and Table S1) with GC being duplicated in the Plasmodium and Eimeria spp., as previously reported (5) (Figure 1B and Table S1). The CDC50 protein family is composed of four members in T. gondii. CDC50.1, CDC50.2 and CDC50.3 are clustered phylogenetically and may have arisen through gene duplication (Figure S1). The presence of three individual genes belonging to this group is only found in coccidians (Figure 1B and Table S1). A single gene assigned to the CDC50.1/2/3 group is present in Cryptosporidia while CDC50.3 is absent in Theileria. In contrast, CDC50.4 is conserved across the entire phylum (Figure 1B, Figure S1 and Table S1).

To determine their localization and scrutinize their function, the genes corresponding to the P4ATPases and CDC50s were C-terminally tagged with 3-HA epitope tags and concomitantly fused to the auxin-inducible degron (mAID) (14) at their endogenous locus via CRISPR-Cas9 genome editing. The resulting mutants were cloned and confirmed by genomic PCR (Figure S2A). Similar to GC and its partner CDC50.1, which have previously been found at the apical cap of the parasites (8), ATP2A, ATP2B, CDC50.2 and CDC50.4 were also found at the apical cap (Figure 1C and 1E). Contrastingly, CDC50.3 localized to the Golgi apparatus (Figure 1E) while ATP7B could only be detected throughout the secretory pathway after tagging with the 
spaghetti monster-HA (SM-HA) (40) (Figure 1D and Figure S2B-C). ATP8 was refractory to genetic modification, which hampered further investigation.

\section{Toxoplasma gondii CDC50.4 forms promiscuously stable heterocomplexes with ATP2A} and ATP2B

Given its conservation across the Apicomplexa, localization and predicted essentiality, we focus our attention on CDC50.4 (28). In order to identify the complex formed by this protein, we performed immunoprecipitation of CDC50.4 coupled with mass spectrometry analysis (Figure S2D). ATP2A and ATP2B were identified as interacting partners of this protein (Figure 2A). Other proteins identified in pull-down are likely contaminants that correspond to highly abundant proteins or are predicted to be implicated in non-related functions. Moreover, endogenous epitope-tagging of ATP2B-Ty in CDC50.4-mAID-HA confirmed co-localization of the two proteins at the apical tip of the parasite (Figure 2B) and pull-down experiments provided further evidence of their stable association (Figure 2C). Compellingly, depletion of CDC50.4 led to a significant decrease in ATP2B protein level (Figure 2D and S2E). Partial colocalization and downregulation of ATP2A upon depletion of CDC50.4 were also shown by immunofluorescence (Figure 2E). In contrast, the absence of CDC50.4 did not impact on the localization of GC-Ty (Figure $2 \mathrm{~F}$ and Figure 2G) and its level of expression (Figure 2H) although it shares the same localization as ATP2B but forms a heterocomplex with CDC50.1 [9]. Taken together, these data strongly indicate that CDC50.4 is forming a complex with ATP2A and ATP2B.

\section{ATP2B-CDC50.4 complex and ATP7B are critical for the survival of Toxoplasma gondii}

The three P-type IV ATPases (ATP2A, ATP2B and ATP7A) and CDC50s (CDC50.2, CDC50.3 and CDC50.4), were C-terminally fused to the auxin-inducible degron (mAID) at the 

213 (Figure 4I and 4J). parasite biology.

endogenous locus (14) and efficiently depleted upon addition of 3-indoleacetic acid (IAA) as confirmed by western blot (Figure 3A and Figure 3B). The relative fitness of each knockdown mutant was assessed by its ability to form plaques of lysis on host cell monolayers (Figure 3C and Figure 3D). Parasite lacking ATP2A and CDC50.2 had no apparent fitness defect, whereas loss ATP2B, ATP7B and CDC50.4 in presence of IAA led to significantly smaller plaques compared to parasites grown in absence of IAA (Figure 3C and Figure 3D). Down-regulation of CDC50.3 resulted in a moderate decrease in fitness (Figure 3C and Figure 3D).

\section{P-type IV ATPases and its CDC50 chaperones play distinct roles in the parasite biology}

To dissect the fitness conferring role of ATP7B depletion, each of the steps of the lytic cycle were examined individually. Parasites depleted in ATP7B are impaired in intracellular growth (Figure S3A) but egress and invade normally (Figure S3B and S3C). Moreover, the organization of intracellular parasites in rosettes was disrupted in the absence of ATP7B (Figure 3SD and 3SE). Deeper characterization would be needed to understand the importance of ATP7B in the

Parasites depleted in either ATP2B or CDC50.4 showed a severe impairment in invasion (Figure 4A) and in egress (Figure 4B), without alteration of intracellular growth (Figure 4C). Importantly, microneme secretion of extracellular parasites depleted in either ATP2B or CDC50.4 triggered by BIPPO (PDE inhibitor which induces the accumulation of cGMP in the cell) was considerably reduced (Figure $4 \mathrm{D}$ and $4 \mathrm{E}$ ). The defect in microneme exocytosis explains the impaired egress and invasion phenotype of ATP2B, suggesting a crucial role of the heterocomplex for the completion of the parasite's lytic cycle. In contrast, depletion of the paralogue protein ATP2A did not affect any steps of the parasite lytic cycle including invasion (Figure 4F), egress (Figure 4G), intracellular growth (Figure 4H) or microneme secretion 
215

216

217

218

219

220

221

222

223

224

\section{ATP2B-CDC50.4, but not ATP2A-CDC50.4, is a phosphatidylserine flippase at the plasma}

\section{membrane}

Anchoring of ATP2A-CDC50.4 and ATP2B-CDC50.4 complex to the parasite plasma membrane was demonstrated by protease protection assay on non-permeabilized parasites. Proteins exposed to the outer leaflet of the plasma membrane are susceptible to cleavage by proteases. Concomitantly, the disappearance of the full length ATP2A, ATP2B and CDC50.4 upon protease treatment demonstrates that these complexes localize to the plasma membrane of the parasite (Figure 5A-5C). The presence of the complexes at the parasite plasma membrane, offers the convenient opportunity to assess its flippase activity using a live cells assay (41) as previously reported in $T$. gondii (8). We focused our investigation on the analysis of phosphatidylserine (PS) since we previously demonstrated that it is the phospholipid that extracellular parasites majorly incorporate into the plasma membrane (8). A bulk timedependent increase in non-quenchable fluorescent analogues of PS was crucially dependent on the presence of ATP2B at the plasma membrane in extracellular (Figure 5D) or intracellular mimicking conditions (Figure S3F), whereas no changes were found upon depletion of ATP2A (Figure 5E). The depletion of CDC50.4 mimicked the effects of the depletion of ATP2B with respect to the bulk PS flipping activity (Figure 5F), in contrast to CDC50.1, which did not affect bulk PS activity upon depletion (Figure 5G).

The impact of ATP2B on microneme secretion implicates the importance of a pool of PS at the inner leaflet of the plasma membrane. Such a pool can be detected using the genetically encoded molecular probe lactadherin C2 domain (Lact-C2) fused to GFP known to bind to PS (42). GFPLact-C2 specifically labelled the parasite periphery (Figure $5 \mathrm{H}$ ) as well as some endomembrane compartments where PS synthesis possibly takes place. Mutation in the specific binding site of Lac-C2 for PS inhibited the plasma membrane localization of the protein (Figure 5I) (42). 
Importantly, no changes were observed in Lac-C2 localization upon depletion of ATP2B (Figure $5 \mathrm{H})$. These results are not surprising since PS is the most abundant anionic phospholipid in eukaryotic membranes (accounting up to $10 \%$ of the total cellular lipids) and it is highly concentrated at the inner leaflet of the plasma membrane (43). Concordantly, due to the high affinity of LacC2 to PS (44), low concentration of PS would be sufficient for its binding and relocalization.

We then reasoned that fluctuation in PS levels would be easier to measure in the outer leaflet of the plasma membrane, where concentration in wild type parasites is low. Compellingly, parasite depleted in ATP2B failed to restore PS asymmetric distribution in natural conditions, leading to an accumulation of PS in the outer leaflet of the plasma membrane that can be detected in extracellular parasites via binding to Annexin V (Figure 5J) (Figure S3G and S3H).

Taken together, these results demonstrate that ATP2B-CDC50.4 complex, but not ATP2A complex, controls flipping of PS at the plasma membrane of $T$. gondii.

\section{DOC2.1 primes micronemes for secretion during egress via its putative calcium binding}

\section{domain}

The contribution of ATP2B-CDC50.4 to microneme exocytosis implies a role for PS in this process. Of relevance, DOC2.1 was reported to participate in microneme secretion (35) and C2 domains are known to bind phospholipids in a calcium-dependent manner $(32,45)$. Aspartic acid (D) residues, previously identified to bind calcium in the C2 domains (46) were found to be conserved in non-degenerated $\mathrm{C} 2$ domain of DOC2.1 (Figure 6A). Although a mutation in DOC2.1 was previously implicated in invasion and egress (35), the protein has never been localized and DOC2.1 gene has not been conditionally down-regulated in T. gondii. C-terminal mAID-HA tagging at the endogenous locus revealed a cytoplasmic punctate pattern of DOC2.1mAID-HA in intracellular or extracellular environments (Figure 6B-C and S4A). Induction of 
microneme secretion (BIPPO or calcium ionophore) did not change the localization of Doc2.1 in extracellular conditions (Figure 6C). DOC2.1-mAID-HA was efficiently depleted upon IAA treatment as shown by western blot (Figure 6D) and conditional depletion of DOC2.1 severely impacted parasite fitness as shown by plaque assay (Figure 6E and Figure 6G). This defect was reflected in parasite inability to egress from host cells (Figure 6H) and to invade new host cells (Figure 6I), without impacting on the intracellular growth (Figure S4D). To assess the importance of the conserved residues putatively involved in calcium binding $\left(\mathrm{D}_{584} \mathrm{D}_{590}\right)$, sitedirected mutagenesis assisted by CRISPR/Cas9 genome editing was performed to generate $\left(\mathrm{D}_{584} \mathrm{D}_{590^{-}}>\mathrm{A}_{584} \mathrm{~A}_{590}\right)$, termed DOC2.1 Mut, and $\left(\mathrm{D}_{584} \mathrm{D}_{590^{-}}>\mathrm{D}_{584} \mathrm{D}_{590}\right)$, termed DOC2 WT, DOC2 transgenic parasites. These mutants were obtained by removing six introns in the endogenous locus of $D O C 2.1$ and replacing the genomic segment with the mutated cDNA fragment of the gene (Figure S4B and S4C). Importantly, the insertion of these mutations (DOC2.1 Mut) did not alter the level of expression of DOC2.1 in the parasites (Figure 6D) but impacted parasite fitness as shown by plaque assay (Figure 6F-G) and parasite egress (Figure $6 \mathrm{H})$. Unexpectedly, only the depletion of DOC2.1 but not the mutation in the putative calcium binding domain led to impaired invasion (Figure 6I) and significant alteration of microneme secretion, following EtOH stimulation of extracellular parasites (Figure 6J). No change in intracellular growth was observed upon mutations in DOC2.1 putative calcium binding (Figure S4E).

\section{Discussion}

Phospholipid asymmetry plays a key role in several indispensable cellular functions including membrane potential (47), receptor based signaling (48) and secretion of vesicles (49). P4ATPases are central flippases that help maintain lipids asymmetry (25). Most P type-IV ATPases usually require CDC50 partners acting as chaperones for correct localization and activity (50), 

312 fitness score deduced from the genome wide analysis (28). In addition, a recent report indicates

and complexes formed between these proteins have been shown to be either highly promiscuous or specific (50). Here we demonstrate that T. gondii encodes for 6 type IV ATPases and 4 CDC50 cofactors with different functions and fitness associated to their deletion. Importantly, some of the data presented here are supported by an overlapping study (30).

\section{P4-ATPases and CDC50 complex formation in Apicomplexa}

Here, we demonstrate that in $T$. gondii, CDC50.4 forms heterocomplexes with ATP2B and ATP2A but not with GC despite sharing a similar localization (8), demonstrating some level of specificity in complex formation independent of protein localization. ATP2B acts as a PS flippase at the plasma membrane which plays a crucial role in microneme exocytosis. The promiscuity of ATP2B substrates was not assessed in this study and would require further analysis. Importantly, a recent report has indicated that $P$. chabaudi recombinant ATP2 is capable of flipping PS and PE (51), which would suggest that $T$. gondii ATP2B or ATP2A would also flip PE. Importantly, the authors of this study identified CDC50A and CDC50B as interactors of ATP2, while CDC50C (homologue of CDC50.4) could not be produced recombinantly (51). This promiscuity of binding remains to be confirmed in vivo since we show here that $T$. gondii ATP2B is incapable of using other CDC50 chaperones to compensate for the lack of CDC50.4.

Assignment of other pairs will await further investigation but ATP7 and CDC50.3 might form another heterocomplex which is absent in Theileria and Cryptosporidia. ATP7 has previously been shown to be essential and localize to the parasite-host interface in Plasmodium parasites (29). Despite not being the main focus of this study, we show here that ATP7B is important for T. gondii intracellular growth while ATP7A has been reported to be dispensable based on its that mutations in the ATP7B are associated with increase resistance of T. gondii to extracellular 
314 environment during in vitro evolution studies (52). The mechanistic details for this emergent

315 resistance are obscure.

316 ATP8 belongs to the class 2 of P4-ATPases, which includes ATP9A and ATP9B in mammals 317 and Neo1p in yeast (50). These proteins do not appear to use CDC50 as $\beta$-subunit which might 318 indicate that apicomplexan ATP8 can function independently of any CDC50 subunit. In yeast 319 and mammals, these proteins translocate PS and affect the Golgi/endosomal system (53) and recycling of endosomes (54). A similar function of ATP8 in PS flipping at the Golgi/endosomal compartment in apicomplexans remains to be assessed.

With the group of alveolates, the apicomplexan parasites as well as some ciliates (55) have directly fused a P4-ATPase with GC catalytic domains to form a large GC protein (5). In $T$. gondii, CDC50.1 was shown to be essential for GC localization and the sensing and integration of external signals, notably phosphatidic acid (8). Plasmodium species possess two genes that harbor a fusion with P4-ATPases GC $\alpha$ and GC $\beta$. Importantly, in P. yoelii, CDC50A has been demonstrated to interact with GC $\beta$ and to be essential for ookinete gliding motility (56). The Plasmodium CDC50A groups with the members of CDC50.3 (Fig S1). On the other hand, the $\beta$-subunit associated to GC $\alpha$ remains to be identified. Plasmodium CDC50A and CDC50B belong to the same phylogenetic subgroup that T. gondii CDC50.1 and CDC50.2, and would be good candidates to bind GCa. Conflictingly, neither of these proteins are essential for Plasmodium yoelii erythrocytic stages (PY17X_0619700 and PY17X_0916600) (57) and, despite discrepancies with a genome wide screening on $P$. falciparum (58), would suggest a possible functional redundancy between $\mathrm{CDC} 50 \mathrm{~A}$ and $\mathrm{CDC} 50 \mathrm{~B}$. 
Plasma membrane asymmetry is an essential need in cell biology (24-26) and flipping of PS is likely to be maintained across the entire lytic cycle of the parasite for survival. Coherently, ATP2B flippase activity is maintained even in intracellular mimicking conditions (Figure S3F). Here, we demonstrate that the first repercussion of the dysregulation of PS asymmetry at the plasma membrane are during egress, invasion and egress. Compellingly, PS in the inner leaflet of the plasma membrane is known to play a critical role in neurotransmitter release (49) and insulin secretion (59) in mammalian cells. Moreover, Candida albicans strains impaired in PS biosynthesis display decreased ability to secrete proteases and phospholipases (60). As in most eukaryotic cells, PS is synthesized at the cytosolic leaflet of the ER in T. gondii (61) and asymmetry is predictably maintained by flippases at the Golgi and plasma membrane $(41,62)$.

Golgi localized PS flippases are key players in exocytic vesicle sorting (63). Once PS reaches the parasite plasma membrane, the ATP2B-CDC50.4 heterocomplex presumably ensures an enrichment of PS at the inner leaflet at the apical tip of the parasites. Any excess of PS at the plasma membrane is rapidly converted into PE as recently demonstrated (30). The disruption of this homeostasis might lead to overall changes in plasma membrane tension, curvature and could also affect the activity of important signaling components (i.e. GC (8-11), PKG (14), PIPLC or DGK1 (21)), explaining the phenotype associated to the depletion of ATP2B-CDC50.4 complex. In addition to the previously reported role of PA (21), PS is a second anionic PL implicated in the docking and/or fusion of the micronemes with the plasma membrane. PA is recognized by $\mathrm{APH}$, an acylated protein at surface of the micronemes (21). A plausible candidate binding to PS could be DOC2.1 (35) or Ferlin 1 (FER1) (37), and since this 359 phospholipid is enriched at the inner leaflet of the plasma membrane (Figure 5H), might contribute to the mechanism of recognition and fusion of micronemes with the plasma membrane for exocytosis, similarly to well-studied mechanisms proposed in model organisms $362(32,64,65)$. 
In addition to its role in exocytosis, 'healthy' exposure of PS has been previously associated to pathogenesis and immune regulation by $T$. gondii (66), as well as other eukaryotic parasites (67, 68). The regulation of PS exposure at the plasma membrane of T. gondii and the role of ATP2BCDC50.4 in this process remain to be investigated. Remarkably, T. gondii is known to secrete a soluble PS decarboxylase which might contribute to a decrease of PS concentration at the outer leaflet of the plasma membrane (69).

A possible implication of ATP2B in phosphatidylthreonine (PT) homeostasis has not been investigated due to the lack of commercially available tools to study this phospholipid. PT was previously described as highly enriched phospholipid in Apicomplexa (61) and was shown to be associated to calcium homeostasis in T. gondii (70). It is possible that the phenotype of ATP2B is, at least partially, associated to an unexplored capacity of ATP2B to translocate PT. On the other hand, PT synthesis was previously shown to impact specifically natural egress (61), while induced egress reminds unaltered (70). In addition, since the lack of PT could not be complemented nor aggravated by excess or reduction of PS (61), it is not likely that PS and PT have redundant functions for secretion. Taken together, this data strongly indicates that lack of PS translocation is the main responsible of the phenotype associated to the depletion of ATP2B-CDC50.4 complex showed here.

\section{Priming of micronemes for secretion}

Although DOC2.1 is essential for microneme secretion during invasion, motility and egress (Figure 6H-J), the putative capacity of its $\mathrm{C} 2$ domain to bind calcium appears to be relevant only during egress (Figure 6H). In mammalian neuron cells, DOC2 has been shown to be important only during asynchronous secretion for neurotransmitters and to be essential for super-priming of synaptic vesicles $(32,71)$. Interestingly, mammalian DOC2 is a small protein mostly composed of two C2 domains while apicomplexan DOC2.1 is a larger protein with 
putative pleiotropic functions. It is plausible that the $\mathrm{C} 2$ domain of DOC2.1, like in mammalian cells, is only important for boosting exocytosis. Such an increase in exocytosis might be particularly relevant during the transition from the intracellular to extracellular phase, where exocytosis is mostly inhibited (i.e. lower production of cGMP or higher PKAc1 activity $(4,8$, $12,72)$ ). After egress, some of this inhibition might be lifted, as previously suggested (4), with the putative calcium binding capacity of DOC2.1 becoming dispensable. In this context, the notion of primed micronemes has been previously suggested $(37,73)$. Importantly, a recent study reports that the overexpression of FER1 led to an accumulation of apical localized micronemes and an increase of constitutive secretion of these organelles (37), suggesting that FER1 would be a component of the priming machinery for microneme secretion.

Overall, this study identified the complex ATP2B-CDC50.4, which is a PS flippase that crucially contributes to motility, invasion and egress. A model by which PS concentration at the inner leaflet of the plasma membrane contribute to microneme docking and exocytosis could imply the participation of DOC2.1. However, this hypothesis awaits further investigations.

\section{Materials and methods}

\section{Bacteria, parasite and host cell culture}

E. coli XL-10 Gold chemo-competent bacteria were used for all recombinant DNA experiments. Parental T. gondii strain Ku80 KO (genotype $\mathrm{RH} \Delta \mathrm{hxgprt} \Delta \mathrm{ku} 80$ ) and parental parasites expressing the Tir1 protein were used in this study (14). T. gondii tachyzoites parental and derivative strains were grown in confluent human foreskin fibroblasts (HFFs) maintained in Dulbecco's Modified Eagle's Medium (DMEM, Gibco) supplemented with 5\% fetal calf serum (FCS), $2 \mathrm{mM}$ glutamine and $25 \mathrm{mg} / \mathrm{ml}$ gentamicin. Depletion of mAID fusion proteins was achieved with $500 \mu \mathrm{M}$ of IAA (14). 


\section{Preparation of T. gondii genomic DNA}

Genomic DNA (gDNA) was prepared from tachyzoites of RH or RH $\Delta \mathrm{Ku} 80$ (here referred as $\Delta \mathrm{ku} 80$ ) strains using the Wizard SV genomic DNA purification (Promega) according to manufacturer's instructions.

\section{DNA vector constructs and transfection}

All primers used in this study are listed in Table S2. Auxin-inducible degradation of ATP2A, ATP2B, ATP7B, CDC50.2, CDC50.3 and CDC50.4 were generated using a PCR fragment encoding the mAID-HA and the HXGPRT cassette produced using the KOD DNA polymerase (Novagen, Merck) with the vector pTUB1:YFP-mAID-3HA as template and the primers indicated in Table S2. A specific sgRNA was generated to introduce a double-stranded break at the $3^{\prime}$ of each gene (primers used to generate the guide are indicated in Table S2).

To replace the genomic locus of DOC2.1 with a cDNA-derived sequence, the C2 domain of the protein was amplified from cDNA (Primers indicated in Table S2). Point mutation was generated in one step using the primers indicated in Table S2. Linearization was performed using ApaI/PacI before transfection. Two guides were used to introduce two double breaks in the genome locus of DOC2.1. Guides were generated using the primers indicated in Table S2.

Parasite transfection and selection of clonal stable lines $T$. gondii tachyzoites were transfected by electroporation as previously described (74). Selection of transgenic parasites were performed either with mycophenolic acid and xanthine for HXGPRT selection (75), pyrimethamine for DHFR selection (76) or chloramphenicol for CAT selection (77). Stable line for all expressing strains were cloned by limited dilution and checked for genomic integration by PCR and analysed by IFA and/or WB. 
438 The monoclonal antibodies against the Ty tag BB2 (1:10 dilution by WB, 1:20 by IFA) (78), 439 actin (1:20 WB) (79), SAG1 (1:20 IFA) (generous gift from J-F. Dubremetz), GRA1 T5-2B4 440 (1:50 WB, 1:100 IFA), GRA3 (1:50 WB, 1:100 IFA) (generous gift from J-F. Dubremetz), 441 MIC2 (1:20 WB, 1:50 IFA) (generous gift from J-F. Dubremetz), MIC3 T4-2F3 (1:20 WB, 1:50 442 IFA), anti-Catalase (1:2000 WB) (80), anti-IMC1 (1:2000 WB, 1:1000 IFA), anti-ARO (1:3000 443 IFA). For western blot analysis, secondary peroxidase-conjugated goat anti-rabbit-IgG, anti444 mouse-IgG antibodies and secondary Alexa-Fluor-680-conjugated goat anti-rabbit IgG 445 antibodies (Thermofisher) were used. For immunofluorescence analysis, the secondary 446 antibodies Alexa-Fluor-488-conjugated goat anti-rabbit IgG antibodies, Alexa-Fluor-488447 conjugated goat anti-mouse-IgG antibodies and Alexa-Fluor-594-conjugated goat anti-mouse448 IgG antibodies (Thermofisher) were used.

Immunofluorescence assay (IFA)

451 HFFs seeded on coverslips in 24-well plates were inoculated with freshly egressed parasites. 452 After $24 \mathrm{~h}$, cells were fixed with 4\% paraformaldehyde (PFA) and $0.005 \%$ glutaraldehyde (GA) 453 in PBS for 10 min and processed as previously described (8). Confocal images were acquired 454 with a Zeiss confocal laser scanning microscope (LSM700 or LSM800) using a PlanApochromat $63 \mathrm{x}$ objective with NA 1.4 at the Bioimaging core facility of the Faculty of Medicine, University of Geneva. Final image analysis and processing was done with Fiji (81).

\section{Western blotting}

459 Freshly egressed parasites were pelleted after complete host cell lysis. SDS-PAGE, wet transfer to nitrocellulose and proteins visualized using ECL system (Amersham Corp) were performed as described previously (8). 


\section{Invasion assay}

473 Freshly egressed parasites were inoculated on coverslips seeded with HFFs monolayers and 474 centrifuged at $1100 \mathrm{x}$ g for $1 \mathrm{~min}$. Invasion was allowed for $20 \mathrm{~min}$ at $37^{\circ} \mathrm{C}+/-\mathrm{ATc}$ prior to 475 fixation with PFA/GA. Extracellular parasites were stained first using monoclonal anti-SAG1

477 formaldehyde/PBS for $7 \mathrm{~min}$ and washed once with PBS. This was followed by 478 permeabilization with $0.2 \%$ Triton/PBS and staining of all parasites with polyclonal anti479 GAP45 Ab. Appropriate secondary Abs were used as previously described. 100 parasites were 480 counted for each experiment, the ratio between red (all) and green (invaded) parasites is presented. Results are presented as mean \pm standard deviation (SD) of three independent

482 biological replicate experiments.

\section{Induced egress assay}

485 Freshly egressed tachyzoites were added to a new monolayer of HFFs, washed after 30 min and grown for $30 \mathrm{~h}$. The infected HFFs were washed once in serum-free DMEM and then incubated

487 with $50 \mu \mathrm{M}$ BIPPO in serum-free DMEM for 7 min at $37^{\circ} \mathrm{C}$. Cells were fixed with PFA/GA 
and processed for IFA using anti-GAP45 Ab. 100 vacuoles were counted per strain and scored as egressed or non-egressed. Results are presented as mean \pm standard deviation (SD) of three independent biological replicate experiments. Control experiment with DMSO showed no egress. For live video microscopy of induced egress, parasites were grown on glass bottom plates seeded with HFFs monolayers for $30 \mathrm{~h}$ at $37^{\circ} \mathrm{C}$ and egress was induced as described 493 above.

\section{Microneme secretion assay}

Microneme secretion assay was performed on freshly egressed parasites, pre-treated 24 or $48 \mathrm{~h}$

497 +/- ATc. Parasites were pelleted at $1000 \mathrm{rpm}$ for $5 \mathrm{~min}$ and resuspended in extracellular (EC) buffer $(142 \mathrm{mM} \mathrm{NaCl}, 5.8 \mathrm{mM} \mathrm{KCl}, 1 \mathrm{mM} \mathrm{MgCl} 2,1 \mathrm{mM} \mathrm{CaCl}$, $5.6 \mathrm{mM}$ glucose, $25 \mathrm{mM}$ HEPES, pH to 7.2 with $\mathrm{NaOH})$. After centrifugation, the pellets were resuspended in $100 \mu \mathrm{L}$ of extracellular (EC) buffer containing $+/-2 \%$ ethanol and incubated for $30 \mathrm{~min}$ at $37^{\circ} \mathrm{C}$. Then, parasites were pelleted at $1000 \mathrm{xg}$ for $10 \mathrm{~min}$ at $4^{\circ} \mathrm{C}$, the supernatant was transferred to a new

Eppendorf tube (the pellet from this step serves as the pellet fraction) and centrifuged again at $2000 \mathrm{x} \mathrm{g}$ for $10 \mathrm{~min}$ at $4^{\circ} \mathrm{C}$. The final supernatant, containing the excreted/secreted antigens (ESA), and pellet fraction were resuspended in SDS loading buffer and boiled prior to immunoblotting.

\section{Flippase assay}

508 NBD-phospholipid incorporation (NBD-PS) was assessed by flow cytometry as described 509 before $(8,41)$. In brief, $5 \times 10^{6}$ extracellular parasites were washed in Hank's balanced salt 510 solution ( $\mathrm{pH} 7.4$ ) containing $1 \mathrm{~g} 1-1$ glucose. Subsequently, $1 \mu \mathrm{M}$ NBD-PS was incubated at 511 room temperature. At the designated time point, $20 \mathrm{mM}$ DPX (p-xylene-bis-pyridinium 512 bromide) was added to quench fluorescence of lipids localized in the outer leaflet. Then, 10,000 
513 cells were analysed with a Gallios (4-laser) cytometer. The mean fluorescence intensities of the 514 cells were calculated.

517 Annexin V (ThermoFisher, 88-8005-72) labelling was performed as indicated by supplier.

518 Briefly, $1 \times 10^{6}$ freshly egressed parasites were resuspended in binding buffer provided by 519 supplier. $5 \mathrm{uL}$ of Annexin V was added for labelling and incubated during 10-15 minutes. Upon 520 washing once, 10,000 cells were analyzed with a Gallios (4-laser) cytometer. The mean 521 fluorescence intensities of the cells were calculated.

In silico analysis of proteins and modelling

524 Sequences of Apicomplexan P-type ATPases and CDC50s were procured from EuPathDB and 525 aligned using MUSCLE sequence alignment software $(82,83)$. The resulting sequence alignment was manually curated utilizing BioEdit

527 (http://www.mbio.ncsu.edu/bioedit/bioedit.html) to edit out uninformative alignment positions. 528 Phylogeny tree was generating utilizing PhyML (84) on the curated MUSCLE alignment, using 529 LG model of amino acids substitution with NNI topology search. Phylogeny.fr (85) platform 530 was utilized for much of the above analysis. All accession numbers are provided in TableS1.

531 C2 domain modelling of TgDOC2.1 was performed using the automated server i-TASSER (86) 532 and visualized using PyMOL (www.pymol.org). Modelling was performed using the residues 533569 to 653 , using the sequences 4ihbA, 3jzyA, 4icw, 5ixcA, 4rj9A, 3pfqA and 5ixcA 534 (Swissmodel templates) with normalized Z-scores of 0.87 to 2.51. Overall model possesses an 535 estimated C-score of -0.41 , TM score of $0.66 \pm 0.13$ and RMSD of $4.4 \pm 2.9 \AA$. 
All data are presented as the mean \pm s.d. of 3 independent biological replicates $(n=3)$, unless otherwise stated in the figure. The mean of each independent biological replicate was generated by counting 100 vacuoles/parasites. All data analyses were carried out using GraphPad Prism. The null hypothesis $(\alpha=0.05)$ was tested using unpaired two-tailed Student's t-tests and significant $P$ values are shown.

\section{Acknowledgments}

This work was supported by the Swiss National Foundation to D.S.-F. (FN3100A0-116722) and by the Scientific \& Technological Cooperation Programme Switzerland-Rio de Janeiro (IZRJZ3_164183). H.B. is the recipient of a Swiss Government Excellence Scholarship with Uruguay. The funders had no role in study design, data collection and analysis, decision to publish, or preparation of the manuscript.

\section{Conflict of interest}

The authors declare no conflict of interest.

\section{References}

1. Kotloff KL, Nataro JP, Blackwelder WC, Nasrin D, Farag TH, Panchalingam S, et al. Burden and aetiology of diarrhoeal disease in infants and young children in developing countries (the Global Enteric Multicenter Study, GEMS): a prospective, case-control study. Lancet. 2013;382(9888):209-22.

2. Morrison WI. Progress towards understanding the immunobiology of Theileria parasites. Parasitology. 2009;136(12):1415-26.

3. Shirley MW, Smith AL, Blake DP. Challenges in the successful control of the avian coccidia. Vaccine. 2007;25(30):5540-7.

4. Bisio H, Soldati-Favre D. Signaling Cascades Governing Entry into and Exit from Host Cells by Toxoplasma gondii. Annu Rev Microbiol. 2019;73:579-99.

5. Baker DA, Drought LG, Flueck C, Nofal SD, Patel A, Penzo M, et al. Cyclic nucleotide signalling in malaria parasites. Open Biol. 2017;7(12).

6. Bullen HE, Bisio H, Soldati-Favre D. The triumvirate of signaling molecules controlling Toxoplasma microneme exocytosis: Cyclic GMP, calcium, and phosphatidic acid. PLoS Pathog. 2019;15(5):e1007670.

7. Dubois DJ, Soldati-Favre D. Biogenesis and secretion of micronemes in Toxoplasma gondii. Cell Microbiol. 2019;21(5):e13018. 
8. Bisio H, Lunghi M, Brochet M, Soldati-Favre D. Phosphatidic acid governs natural egress in Toxoplasma gondii via a guanylate cyclase receptor platform. Nat Microbiol. 2019;4(3):4208.

9. Brown KM, Sibley LD. Essential cGMP Signaling in Toxoplasma Is Initiated by a Hybrid P-Type ATPase-Guanylate Cyclase. Cell Host Microbe. 2018;24(6):804-16 e6.

10. Gunay-Esiyok O, Scheib U, Noll M, Gupta N. An unusual and vital protein with guanylate cyclase and P4-ATPase domains in a pathogenic protist. Life Sci Alliance. 2019;2(3).

11. Yang L, Uboldi AD, Seizova S, Wilde ML, Coffey MJ, Katris NJ, et al. An apically located hybrid guanylate cyclase-ATPase is critical for the initiation of $\mathrm{Ca}(2+)$ signaling and motility in Toxoplasma gondii. J Biol Chem. 2019;294(22):8959-72.

12. Jia Y, Marq JB, Bisio H, Jacot D, Mueller C, Yu L, et al. Crosstalk between PKA and PKG controls pH-dependent host cell egress of Toxoplasma gondii. EMBO J. 2017;36(21):3250-67. 13. Uboldi AD, Wilde ML, McRae EA, Stewart RJ, Dagley LF, Yang L, et al. Protein kinase A negatively regulates Ca2+ signalling in Toxoplasma gondii. PLoS Biol. 2018;16(9):e2005642. 14. Brown KM, Long S, Sibley LD. Plasma Membrane Association by N-Acylation Governs PKG Function in Toxoplasma gondii. mBio. 2017;8(3).

15. Brochet M, Collins MO, Smith TK, Thompson E, Sebastian S, Volkmann K, et al. Phosphoinositide metabolism links cGMP-dependent protein kinase $\mathrm{G}$ to essential $\mathrm{Ca}(2)(+)$ signals at key decision points in the life cycle of malaria parasites. PLoS Biol. 2014;12(3):e1001806.

16. Lourido S, Moreno SN. The calcium signaling toolkit of the Apicomplexan parasites Toxoplasma gondii and Plasmodium spp. Cell Calcium. 2015;57(3):186-93.

17. Lourido S, Shuman J, Zhang C, Shokat KM, Hui R, Sibley LD. Calcium-dependent protein kinase 1 is an essential regulator of exocytosis in Toxoplasma. Nature. 2010;465(7296):359-62. 18. Lourido S, Tang K, Sibley LD. Distinct signalling pathways control Toxoplasma egress and host-cell invasion. EMBO J. 2012;31(24):4524-34.

19. Tosetti N, Dos Santos Pacheco N, Soldati-Favre D, Jacot D. Three F-actin assembly centers regulate organelle inheritance, cell-cell communication and motility in Toxoplasma gondii. Elife. 2019;8.

20. Frenal K, Dubremetz JF, Lebrun M, Soldati-Favre D. Gliding motility powers invasion and egress in Apicomplexa. Nat Rev Microbiol. 2017;15(11):645-60.

21. Bullen HE, Jia Y, Yamaryo-Botte Y, Bisio H, Zhang O, Jemelin NK, et al. Phosphatidic Acid-Mediated Signaling Regulates Microneme Secretion in Toxoplasma. Cell Host Microbe. 2016;19(3):349-60.

22. Kumar Sah R, Garg S, Dangi P, Ponnusamy K, Singh S. Phosphatidic acid homeostasis regulated by a type- 2 phosphatidic acid phosphatase represents a novel druggable target in malaria intervention. Cell Death Discov. 2019;5:107.

23. Darvill N, Dubois DJ, Rouse SL, Hammoudi PM, Blake T, Benjamin S, et al. Structural Basis of Phosphatidic Acid Sensing by APH in Apicomplexan Parasites. Structure. 2018;26(8):1059-71 e6.

24. McMahon HT, Boucrot E. Membrane curvature at a glance. J Cell Sci. 2015;128(6):106570 .

25. Daleke DL. Phospholipid flippases. J Biol Chem. 2007;282(2):821-5.

26. Roland BP, Graham TR. Decoding P4-ATPase substrate interactions. Crit Rev Biochem Mol Biol. 2016;51(6):513-27.

27. Hiraizumi M, Yamashita K, Nishizawa T, Nureki O. Cryo-EM structures capture the transport cycle of the P4-ATPase flippase. Science. 2019;365(6458):1149-55.

28. Sidik SM, Huet D, Ganesan SM, Huynh MH, Wang T, Nasamu AS, et al. A Genome-wide CRISPR Screen in Toxoplasma Identifies Essential Apicomplexan Genes. Cell. 2016;166(6):1423-35 e12. 
29. Kenthirapalan S, Waters AP, Matuschewski K, Kooij TW. Functional profiles of orphan membrane transporters in the life cycle of the malaria parasite. Nat Commun. 2016;7:10519. 30. Chen K, Gunay-Esiyok O, Klingeberg M, Marquardt S, Pomorski TG, Gupta N. Aminoglycerophospholipid flipping and P4-ATPases in Toxoplasma gondii. J Biol Chem. 2021:100315.

31. Sudhof TC, Rizo J. Synaptic vesicle exocytosis. Cold Spring Harb Perspect Biol. 2011;3(12).

32. Yao J, Gaffaney JD, Kwon SE, Chapman ER. Doc2 is a $\mathrm{Ca} 2+$ sensor required for asynchronous neurotransmitter release. Cell. 2011;147(3):666-77.

33. Wang S, Li Y, Ma C. Synaptotagmin-1 C2B domain interacts simultaneously with SNAREs and membranes to promote membrane fusion. Elife. 2016;5.

34. Bisio H, Chaabene RB, Sabitzki R, Maco B, Marq JB, Gilberger TW, et al. The ZIP Code of Vesicle Trafficking in Apicomplexa: SEC1/Munc18 and SNARE Proteins. mBio. 2020;11(5).

35. Farrell A, Thirugnanam S, Lorestani A, Dvorin JD, Eidell KP, Ferguson DJ, et al. A DOC2 protein identified by mutational profiling is essential for apicomplexan parasite exocytosis. Science. 2012;335(6065):218-21.

36. Obrova K, Cyrklaff M, Frank R, Mair GR, Mueller AK. Transmission of the malaria parasite requires ferlin for gamete egress from the red blood cell. Cell Microbiol. 2019;21(5):e12999.

37. Tagoe DNA, Drozda AA, Coppens I, Coleman BI, Gubbels M-J. <em>Toxoplasma</em> ferlin1 is a versatile and dynamic mediator of microneme trafficking and secretion. bioRxiv. 2020:2020.04.27.063628.

38. Tagoe DNA, Drozda AA, Falco JA, Bechtel TJ, Weerapana E, Gubbels M-J. Ferlins and TgDOC2 in Toxoplasma Microneme, Rhoptry and Dense Granule Secretion. Life. 2021;11(3):217.

39. Martin RE, Ginsburg H, Kirk K. Membrane transport proteins of the malaria parasite. Mol Microbiol. 2009;74(3):519-28.

40. Hortua Triana MA, Marquez-Nogueras KM, Chang L, Stasic AJ, Li C, Spiegel KA, et al. Tagging of Weakly Expressed Toxoplasma gondii Calcium-Related Genes with High-Affinity Tags. J Eukaryot Microbiol. 2018;65(5):709-21.

41. Takatsu H, Tanaka G, Segawa K, Suzuki J, Nagata S, Nakayama K, et al. Phospholipid flippase activities and substrate specificities of human type IV P-type ATPases localized to the plasma membrane. J Biol Chem. 2014;289(48):33543-56.

42. Yeung T, Gilbert GE, Shi J, Silvius J, Kapus A, Grinstein S. Membrane phosphatidylserine regulates surface charge and protein localization. Science. 2008;319(5860):210-3.

43. Kay JG, Faim GD. Distribution, dynamics and functional roles of phosphatidylserine within the cell. Cell Commun Signal. 2019;17(126).

44. Shao C, Novakovic VA, Head JF, Seaton BA, Gilbert GE. Crystal structure of lactadherin $\mathrm{C} 2$ domain at $1.7 \mathrm{~A}^{\circ}$ resolution with mutational and computational analyses of its membranebinding motif. Journal of Biological Chemistry. 2008;283(11):7230-7241.

45. Jean S, Zapata-Jenks MA, Farley JM, Tracy E, Mayer DC. Plasmodium falciparum double C2 domain protein, PfDOC2, binds to calcium when associated with membranes. Exp Parasitol. 2014;144:91-5.

46. Corbalan-Garcia S, Gomez-Fernandez JC. Signaling through C2 domains: More than one lipid target. Bba-Biomembranes. 2014;1838(6):1536-47.

47. Gurtovenko AA, Vattulainen I. Membrane potential and electrostatics of phospholipid bilayers with asymmetric transmembrane distribution of anionic lipids. J Phys Chem B. 2008;112(15):4629-34. 
48. Kim HY, Huang B, Akbar M, Kevala K. Phosphatidylserine is a critical modulator for Akt activation. Faseb J. 2011;25.

49. Kim HY, Huang BX, Spector AA. Phosphatidylserine in the brain: metabolism and function. Prog Lipid Res. 2014;56:1-18. 50. Andersen JP, Vestergaard AL, Mikkelsen SA, Mogensen LS, Chalat M, Molday RS. P4ATPases as Phospholipid Flippases-Structure, Function, and Enigmas. Front Physiol. 2016;7. 51. Lamy A, Macarini-Bruzaferro E, Dieudonne T, Peralvarez-Marin A, Lenoir G, Montigny C, et al. ATP2, The essential P4-ATPase of malaria parasites, catalyzes lipid-stimulated ATP hydrolysis in complex with a Cdc50 beta-subunit. Emerg Microbes Infect. 2021;10(1):132-47. 52. Primo VA, Rezvani Y, Farrell A, Vajdi A, Marth GT, Zarringhalam K, et al. The extracellular milieu of <em>Toxoplasma</em>'s lytic cycle drives lab-adaptation and promotes changes in lipid metabolism primarily driven by transcriptional reprogramming. bioRxiv. 2021:2021.03.09.434570.

53. Wicky S, Schwarz H, Singer-Kruger B. Molecular interactions of yeast Neo1p, an essential member of the Drs2 family of aminophospholipid translocases, and its role in membrane trafficking within the endomembrane system. Mol Cell Biol. 2004;24(17):7402-18.

54. Tanaka Y, Ono N, Shima T, Tanaka G, Katoh Y, Nakayama K, et al. The phospholipid flippase ATP9A is required for the recycling pathway from the endosomes to the plasma membrane. Mol Biol Cell. 2016;27(24):3883-93.

55. Linder JU, Hoffmann T, Kurz U, Schultz JE. A guanylyl cyclase from Paramecium with 22 transmembrane spans. Expression of the catalytic domains and formation of chimeras with the catalytic domains of mammalian adenylyl cyclases. J Biol Chem. 2000;275(15):11235-40.

56. Gao H, Yang Z, Wang X, Qian P, Hong R, Chen X, et al. ISP1-Anchored Polarization of GCbeta/CDC50A Complex Initiates Malaria Ookinete Gliding Motility. Curr Biol. 2018;28(17):2763-76 e6.

57. Jiang Y, Wei J, Cui H, Liu C, Zhi Y, Jiang Z, et al. An intracellular membrane protein GEP1 regulates xanthurenic acid induced gametogenesis of malaria parasites. Nat Commun. 2020;11(1):1764.

58. Zhang M, Wang C, Otto TD, Oberstaller J, Liao X, Adapa SR, et al. Uncovering the essential genes of the human malaria parasite Plasmodium falciparum by saturation mutagenesis. Science. 2018;360(6388):eaap7847.

59. Ansari IU, Longacre MJ, Paulusma CC, Stoker SW, Kendrick MA, MacDonald MJ. Characterization of P4 ATPase Phospholipid Translocases (Flippases) in Human and Rat Pancreatic Beta Cells: THEIR GENE SILENCING INHIBITS INSULIN SECRETION. J Biol Chem. 2015;290(38):23110-23.

60. Wolf JM, Espadas J, Luque-Garcia J, Reynolds T, Casadevall A. Lipid Biosynthetic Genes Affect Candida albicans Extracellular Vesicle Morphology, Cargo, and Immunostimulatory Properties. Eukaryot Cell. 2015;14(8):745-54.

61. Arroyo-Olarte RD, Brouwers JF, Kuchipudi A, Helms JB, Biswas A, Dunay IR, et al. Phosphatidylthreonine and Lipid-Mediated Control of Parasite Virulence. PLoS Biol. 2015;13(11):e1002288.

63. Hankins HM, Sere YY, Diab NS, Menon AK, Graham TR. Phosphatidylserine translocation at the yeast trans-Golgi network regulates protein sorting into exocytic vesicles. Mol Biol Cell. 2015;26(25):4674-85.

64. Brouwer I, Giniatullina A, Laurens N, van Weering JRT, Bald D, Wuite GJL, et al. Direct quantitative detection of Doc2b-induced hemifusion in optically trapped membranes. Nat Commun. 2015;6(8387).

65. Orita S, Sasaki T, Naito A, Komuro R, Ohtsuka T, Maeda M, et al. Doc2: A Novel Brain Protein Having Two Repeated C2-like Domains. Biochem and Biophysical Research Commun. $1995 ; 206(2)$. 
66. dos Santos TAT, Portes JD, Damasceno-Sa JC, Caldas LA, de Souza W, DaMatta RA, et al. Phosphatidylserine Exposure by Toxoplasma gondii Is Fundamental to Balance the Immune Response Granting Survival of the Parasite and of the Host. Plos One. 2011;6(11). 67. DaMatta RA, Seabra SH, Deolindo P, Arnholdt ACV, Manhaes L, Goldenberg S, et al. Trypanosoma cruzi exposes phosphatidylserine as an evasion mechanism. Fems Microbiol Lett. 2007;266(1):29-33.

68. Tripathi A, Gupta CM. Transbilayer translocation of membrane phosphatidylserine and its role in macrophage invasion in Leishmania promastigotes. Mol Biochem Parasit. 2003;128(1):1-9.

69. Gupta N, Hartmann A, Lucius R, Voelker DR. The obligate intracellular parasite Toxoplasma gondii secretes a soluble phosphatidylserine decarboxylase. J Biol Chem. 2012;287(27):22938-47.

70. Kuchipudi A, Arroyo-Olarte RD, Hoffmann F, Brinkmann V, Gupta N. Optogenetic monitoring identifies phosphatidylthreonine-regulated calcium homeostasis in Toxoplasma gondii. Microb Cell. 2016;3(5):215-23.

71. Xue R, Ruhl DA, Briguglio JS, Figueroa AG, Pearce RA, Chapman ER. Doc2-mediated superpriming supports synaptic augmentation. Proc Natl Acad Sci U S A. 2018;115(24):E5605E13.

72. Burda PC, Bisio H, Marq JB, Soldati-Favre D, Heussler VT. CRISPR/Cas9-Based Knockout of GNAQ Reveals Differences in Host Cell Signaling Necessary for Egress of Apicomplexan Parasites. mSphere. 2020;5(6).

73. Sakura T, Sindikubwabo F, Oesterlin LK, Bousquet H, Slomianny C, Hakimi MA, et al. A Critical Role for Toxoplasma gondii Vacuolar Protein Sorting VPS9 in Secretory Organelle Biogenesis and Host Infection. Sci Rep. 2016;6:38842.

74. Soldati D, Boothroyd JC. Transient transfection and expression in the obligate intracellular parasite Toxoplasma gondii. Science. 1993;260(5106):349-52.

75. Donald RG, Carter D, Ullman B, Roos DS. Insertional tagging, cloning, and expression of the Toxoplasma gondii hypoxanthine-xanthine-guanine phosphoribosyltransferase gene. Use as a selectable marker for stable transformation. J Biol Chem. 1996;271(24):14010-9.

76. Donald RG, Roos DS. Stable molecular transformation of Toxoplasma gondii: a selectable dihydrofolate reductase-thymidylate synthase marker based on drug-resistance mutations in malaria. Proc Natl Acad Sci U S A. 1993;90(24):11703-7.

77. Kim K, Soldati D, Boothroyd JC. Gene replacement in Toxoplasma gondii with chloramphenicol acetyltransferase as selectable marker. Science. 1993;262(5135):911-4.

78. Bastin P, Bagherzadeh Z, Matthews KR, Gull K. A novel epitope tag system to study protein targeting and organelle biogenesis in Trypanosoma brucei. Mol Biochem Parasitol. 1996;77(2):235-9.

79. Herm-Gotz A, Weiss S, Stratmann R, Fujita-Becker S, Ruff C, Meyhofer E, et al. Toxoplasma gondii myosin A and its light chain: a fast, single-headed, plus-end-directed motor. EMBO J. 2002;21(9):2149-58.

80. Ding M, Clayton C, Soldati D. Toxoplasma gondii catalase: are there peroxisomes in Toxoplasma? J Cell Sci. 2000;113(13):2409-19.

81. Schindelin J, Arganda-Carreras I, Frise E, Kaynig V, Longair M, Pietzsch T, et al. Fiji: an open-source platform for biological-image analysis. Nat Methods. 2012;9(7):676-82.

82. Edgar RC. MUSCLE: a multiple sequence alignment method with reduced time and space complexity. BMC Bioinformatics. 2004;5:113.

83. Edgar RC. MUSCLE: multiple sequence alignment with high accuracy and high throughput. Nucleic Acids Res. 2004;32(5):1792-7. 
84. Dereeper A, Guignon V, Blanc G, Audic S, Buffet S, Chevenet F, et al. Phylogeny.fr: robust phylogenetic analysis for the non-specialist. Nucleic Acids Res. 2008;36(Web Server issue):W465-9.

85. Yang J, Yan R, Roy A, Xu D, Poisson J, Zhang Y. The I-TASSER Suite: protein structure and function prediction. Nat Methods. 2015;12(1):7-8.

\section{Figure legends}

Figure 1. Members of the Apicomplexa phylum encode a plethora of P4-ATPases and

\section{CDC50 cofactor at different parasite locations}

(A) Schematic representation of the domain architecture of the P4-ATPase-CDC50 heterocomplex. (B) Conservation of P4-ATPases and CDC50 cofactors across the Apicomplexa phylum. Blue circle: Absent in Theileria. Eimeria possesses two genes belonging to the CDC50.1/CDC50.2 subgroups but direct homology could not be deducted by blast analysis. See Figure S1. Fitness scores associated to gene disruption in T. gondii are obtained from (28). CM: Cyst-forming. PM: plasma membrane. G: Golgi apparatus. A: Apical. S.p: Secretory pathway. N.a: Not assessed. Accession numbers of all putative orthologs genes are included in Table S1. (C) Indirect immunofluorescence assay (IFA) of intracellular ATP2A-mAID-HA and ATP2B-mAID-HA parasites. GAP45: parasite periphery. (D) IFA of intracellular ATP7B-SMHA parasites. Actin: Parasite cytosol. (E) IFA of intracellular CDC50.2, CDC50.3 and CDC50.4-mAID-HA parasites. GAP45: parasite pellicle. Cam-Like protein: Golgi apparatus. The scale bars for the immunofluorescence images are $7 \mu \mathrm{M}$, unless otherwise indicated.

\section{Figure 2. ATP2B and CDC50.4 form an heterocomplex}

(A) Gene ID and number of unique peptides identified as putative interactors of CDC50.4 upon coimmunoprecipitation and mass spectrometry analysis (B) IFA of intracellular ATP2BTy/CDC50.4-mAID-HA parasites. (C) Western blot of immunoprecipitation with anti-Ty from ATP2B-Ty/CDC50.4-mAID-HA lysate showing that ATP2B-Ty is associated with CDC50.4- 
mAID-HA. (D) Western blot of lysates ATP2B-Ty/CDC50.4-mAID-HA parasites treated with or without IAA for 24 hours. Actin: loading control. (E) IFA of intracellular RH ATP2ATy/CDC50.4-mAID-HA parasites with or without IAA. DAPI is shown in presence of IAA in order to demonstrate the presence of parasites. (F) IFA of intracellular RH GC-Ty/CDC50.4mAID-HA parasites with or without IAA. (G) Quantification of representative pictures in (F). The ratio between the intensity of fluorescence at the basal pole versus the apical pole of the parasite is shown. Approximately 100 vacuoles were quantified. $(\mathrm{H})$ Western blot of lysates GC-Ty/CDC50.4-mAID-HA parasites treated with or without IAA for 24 hours. Catalase: loading control. The scale bars for the immunofluorescence images are $7 \mu \mathrm{M}$, unless otherwise indicated.

Figure 3. Fitness conferring and dispensable P-type IV ATPases and CDC50 subunits are encoded in the $T$. gondii genome

(A) Western blot analysis of ATP2A-mAID, ATP2B-mAID and ATP7B-mAID regulation with IAA treatment for 24 hours. Actin: loading control. P: parental strain (Tir1 strain) (B) Western blot of lysates from CDC50.2-4-mAID parasites treated with or without IAA for 24 hours. Actin: loading control. (C) Images of plaques formed by RH Tir1 parental strain, ATP2A, ATP2B, ATP7B, CDC50.2, CDC50.3 and CDC50.4-mAID-HA lines on HFF monolayers with or without IAA treatment. (D) Quantification of plaque size relative to the parental control (Tir1), mean +/- SD of 1 representative experiment. Each parasite line was analysed individually for statistical significance using an unpaired t test. P values: $* * * *=<0.0001, *=<0.05$.

\section{Figure 4. ATP2B-CDC50.4 heterocomplex facilitates microneme secretion}

(A) Invasion assay of Tir1 parental strain, ATP2B-mAID-HA and CDC50.4-mAID-HA parasites treated with or without IAA for 24 hours. Data represents mean +/- SD of three 
822 independent experiments. (B) Egress assay of Tir1 parental strain, ATP2B-mAID-HA and CDC50.4-mAID-HA parasites grown for 30 hours treated with or without IAA. Egress was induced with BIPPO (PDE inhibitor which induces the accumulation of cGMP in the cell) or DMSO for 7 minutes. The percentage of egress (lysed vacuoles) is shown as means +/- SD of 3 independent replicates. (C) Parasites lacking ATP2B or CDC50.4 are not impaired in intracellular replication. Error bars represent +/- SD from three independent experiments. (D) Microneme secretion of extracellular of Tir1 parental strain, ATP2B-mAID-HA and CDC50.4mAID-HA parasites stimulated with or without BIPPO after having been treated or not with IAA for 24 hours. ESA (excreted-secreted antigens) and pellet fractions are shown. MIC2: microneme ESA. GRA1: dense granule ESA. Catalase: lysis control. Relative ratio of MIC2 secretion compared to Tir 1 -IAA parental control +/- SD of 3 independent replicates is shown in (E). (F) Invasion assay of Tir1 parental strain and ATP2A-mAID-HA parasites treated with or without IAA for 24 hours. Data represents mean $+/-$ SD of three independent experiments. (G) Egress assay of Tir1 parental strain and ATP2A-mAID-HA parasites grown for 30 hours treated with or without IAA. Egress was induced with BIPPO (PDE inhibitor which induces the accumulation of cGMP in the cell) or DMSO for 7 minutes. The percentage of egress (lysed vacuoles) is shown as means $+/-$ SD of 3 independent replicates. (H) Parasites lacking ATP2A are not impaired in intracellular replication. Error bars represent $+/$ - SD from three independent experiments. (I) Microneme secretion of extracellular of Tir1 parental strain and ATP2AmAID-HA parasites stimulated with or without BIPPO after having been treated or not with IAA for 24 hours. ESA and pellet fractions are shown. MIC2: microneme ESA. GRA1: dense granule ESA. Catalase: lysis control. Relative ratio of MIC2 secretion compared to Tir1 -IAA parental control +/- SD of 3 independent replicates is shown in $(\mathrm{J})$. Each parasite line was analysed individually for statistical significance using an unpaired $\mathrm{t}$ test. $\mathrm{P}$ values: $* * * *=$ $<0.0001, *=<0.05$. 
Figure 5. ATP2A and ATP2B are phospholipid specific flippases at the parasite plasma

\section{membrane}

850

851 residues ( $\mathrm{D}$ sites) mutated in this study are shown in the inset. Conserved Asp residues

871 putatively involved in Calcium binding are shown in an alignment (highlighted in red). (B) IF 
microscopy of intracellular DOC2.1-mAID-HA parasites. Actin: Cytosol. (C) IF microscopy of

873 extracellular DOC2.1-mAID-HA parasites in presence or absence of different inducers of 874 microneme secretion. Actin: Cytosol. (D)Western blot of lysates from Tir1 parental strain, DOC2.1-mAID-HA and DOC2.1 C2 domain mutant parasites treated with or without IAA for 24 hours. MIC2: loading control. (E) Images of plaques formed by Tir1 parental strain, DOC2.1-mAID-HA lines on HFF monolayers with or without IAA treatment. Quantification of plaque size relative to the parental control (Tir1), mean +/- SD of 1 representative experiment, are also shown in (G). (F) Images of plaques formed by DOC2.1-mAID-HA parental and DOC2.1 C2 domain mutant parasites on HFF monolayers. Quantification of plaque size relative to the parental control (Doc2.1-mAID-HA), mean +/- SD of 1 representative experiment, are also shown in $(\mathrm{G}) .(\mathrm{H})$ Egress assay of RH Tir1 parental, DOC2.1-mAID-HA and DOC2.1 C2 domain mutant parasites grown for 30 hours treated with or without IAA. Egress was induced with BIPPO (PDE inhibitor which induces the accumulation of cGMP in the cell) or DMSO for 7 minutes. Percentage of egressed vacuoles is shown as means +/- SD of 3 independent replicates. (I) Invasion assay of RH Tir1 parental, DOC2.1-mAID-HA and DOC2.1 C2 domain mutant parasites treated with or without IAA for 24 hours. Data represents mean +/- SD of three independent experiments. (J) Microneme secretion of extracellular of RH Tir1 parental, DOC2.1-mAID-HA and DOC2.1 C2 domain mutant parasites stimulated with or without EtOH following 24 hours pre-treatment with or without IAA. ESA and pellet fractions are shown. MIC2: microneme ESA. GRA1: dense granule ESA. Catalase: lysis control. Each parasite line was analysed individually for statistical significance using an unpaired $\mathrm{t}$ test. $\mathrm{P}$ values: $* * * *=$ $<0.0001, *=<0.05$. The scale bars for the immunofluorescence images are $7 \mu \mathrm{M}$, unless otherwise indicated.

\section{Supplementary Figure 1}


897 (A) Apicomplexan CDC50s cluster into 2 phylogenetic groups. An unrooted maximum likelihood tree of apicomplexan ASPs was generated using PhyML v3.0, using WAG model of

899

900

901

902

\section{Supplementary Figure 3}

3 independent replicates. (C) Invasion assay of Tir1 parental strain and ATP7B-mAID-HA

(A) Parasites lacking ATP7B display a delay in intracellular replication. Error bars represent \pm SD from three independent experiments. (B) Egress assay of Tir1 parental strain and ATP7BmAID-HA parasites grown for $30 \mathrm{hs}$ treated with or without IAA. Egress was induced with BIPPO or DMSO for 7 minutes. Percentage of egressed vacuoles is shown as means $+/-$ SD of parasites treated with or without IAA for 24 hours. Data represents mean +/- SD. (D)

919 Representative images of vacuole organization in parasites depleted (or not) of ATP7B. Quantification is shown in (E). (F) Flow cytometry measurement of residual fluorescence upon addition of DPX to NBD-PS incubated extracellular ATP2B-mAID in intracellular buffer. 
922 Parasites were mechanically released from intracellular condition to avoid activation of egress

923 signalling. (G-H) Histograms corresponding to one experiment of Annexin V binding to 924 parasites are shown in $(\mathrm{G})$ and representative images in $(\mathrm{H})$. The scale bars for the

925 immunofluorescence images are $7 \mu \mathrm{M}$, unless otherwise indicated.

927 Supplementary Figure 4

928 (A) PCR demonstrates correct integration of DOC2.1-mAID. Primers used are listed in Table 929 S2. (B) PCR demonstrates correct removal of introns for gene locus modification. Primers used 930 are listed in Table S2. (C) Sequencing of mutant DNA demonstrates correct incorporation of 931 point mutations in the doc2.1 locus. (D) Parasites lacking DOC2.1 are not impaired in 932 intracellular replication. Error bars represent \pm SD from three independent experiments. (E) 933 Parasites mutated on the putative calcium binding of DOC2.1 are not impaired in intracellular 934 replication. Error bars represent \pm SD from three independent experiments.

\section{Supplementary Tables}

937 Table S1. Gene accession numbers of the homologs of the studied genes within the 938 Apicomplexa phylum.

Table S2. Oligonucleotide sequences used in this study. 
Figure 1

A

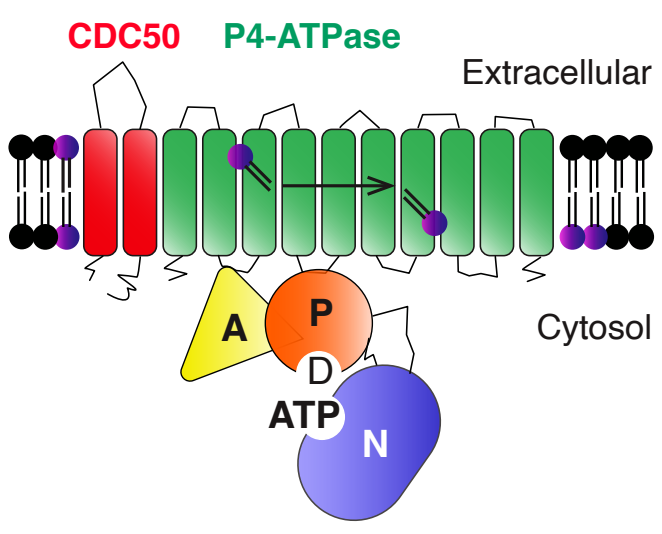

B

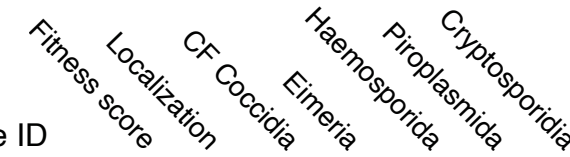

\begin{tabular}{|c|c|c|c|c|c|c|c|}
\hline ATP2A & TGME49_216380 & -0.4 & A & & & & \\
\hline ATP2B & TGME49_247690 & -1.5 & PM & & & & \\
\hline ATP7A & TGME49_257720 & -0.4 & n.a. & & & & \\
\hline ATP7B & TGME49_245510 & -3.8 & $\mathrm{~A} / \mathrm{sp}$ & & & & \\
\hline ATP8 & TGME49_224190 & -5.2 & n.a. & & & & \\
\hline$G C(a)$ & TGME49_254370 & -3.6 & PM & & & & \\
\hline \multicolumn{8}{|l|}{ GC $\beta$} \\
\hline CDC50.1 & TGME49_230820 & -3.2 & PM & & & & \\
\hline CDC50.2 & TGME49_232040 & 0.2 & A & & & & \\
\hline CDC50.3 & TGME49_239540 & -2.7 & $\mathrm{G}$ & & & & \\
\hline CDC50.4 & TGME49_247380 & -3.9 & PM & & & & \\
\hline
\end{tabular}

E

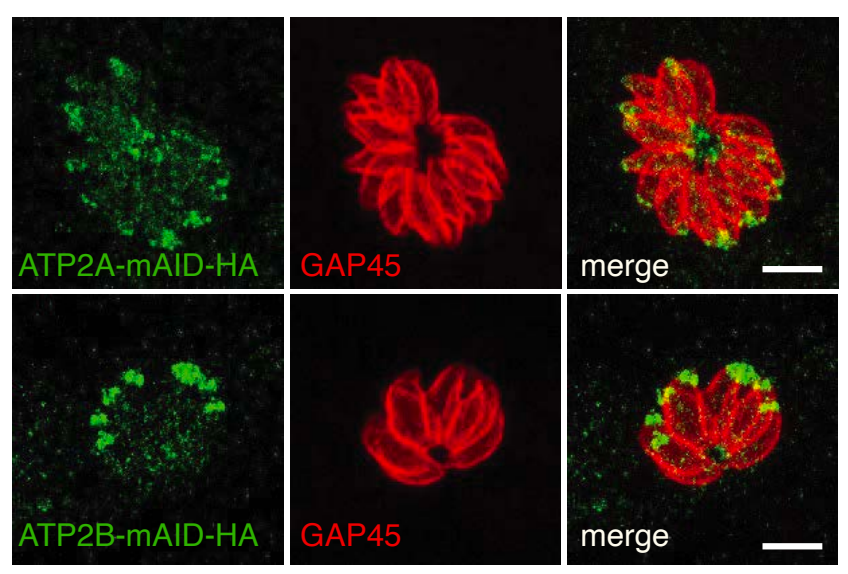

D

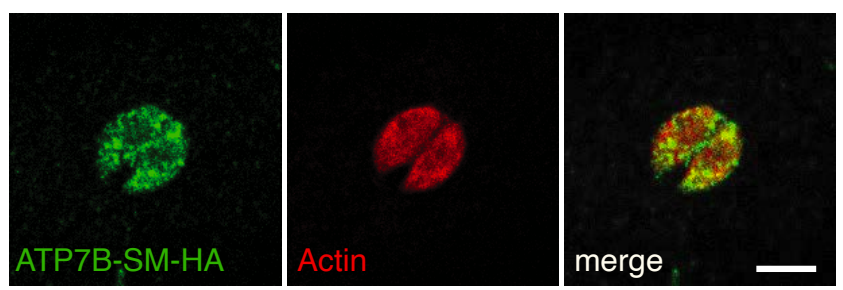

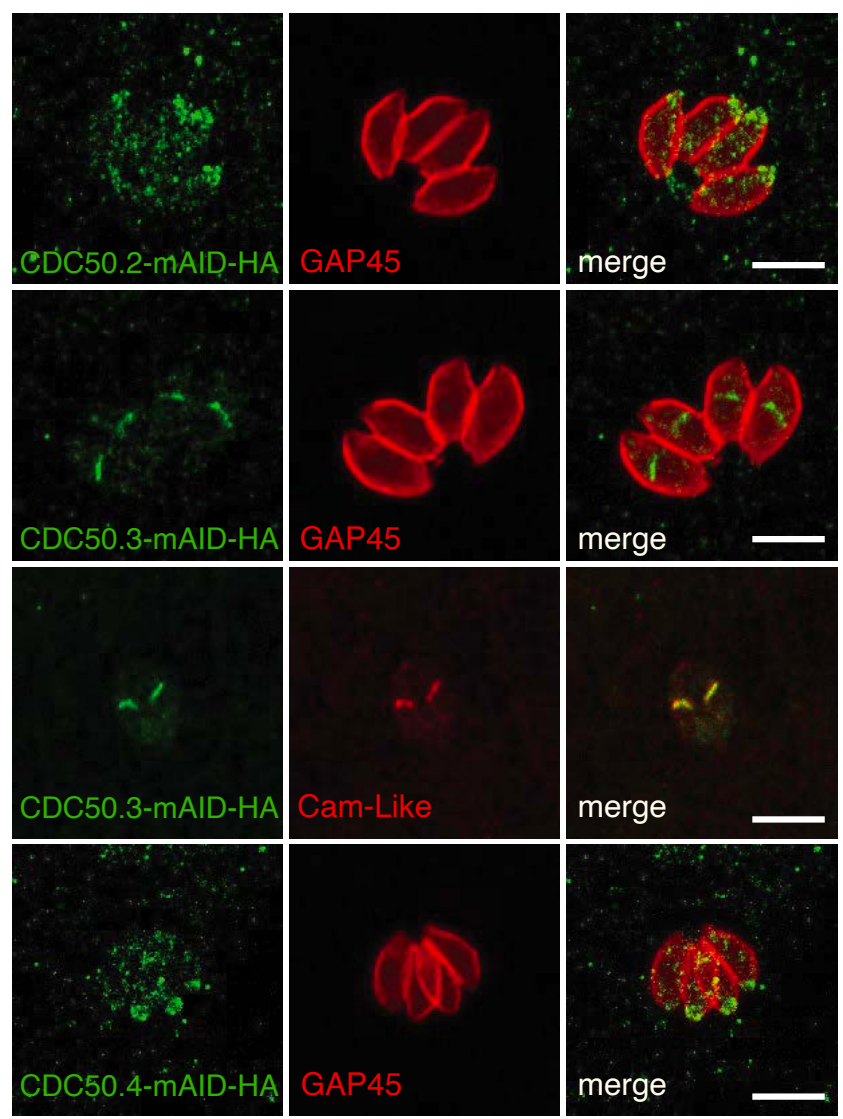


Figure 2

A

\begin{tabular}{c|cc} 
Gene ID & Gene Description & Peptide \\
Count \\
\hline TGME49_247690 & ATP2B & $\mathbf{4 5}$ \\
\hline TGME49_247380 & CDC50.4 & $\mathbf{2 6}$ \\
\hline TGME49_264660 & SAG-related sequence SRS44 & $\mathbf{1 6}$ \\
\hline TGME49_216380 & ATP2A & $\mathbf{1 4}$ \\
\hline TGME49_308090 & ROP5 & $\mathbf{1 0}$ \\
\hline TGME49_411430 & ROP5 & $\mathbf{1 0}$ \\
\hline TGME49_273760 & HSP70 & $\mathbf{8}$ \\
\hline TGME49_241170 & hypothetical protein & $\mathbf{8}$ \\
\hline TGME49_213950 & hypothetical protein & $\mathbf{5}$ \\
\hline TGME49_233100 & SPFH domain & $\mathbf{5}$ \\
\hline TGME49_205250 & ROP18 & $\mathbf{4}$ \\
\hline TGME49_262960 & U1 snRNP-associated protein Usp106 & $\mathbf{4}$ \\
\hline TGME49_209030 & Actin (ACT1) & $\mathbf{4}$ \\
\hline TGME49_270240 & MAG1 & $\mathbf{4}$ \\
\hline
\end{tabular}

E

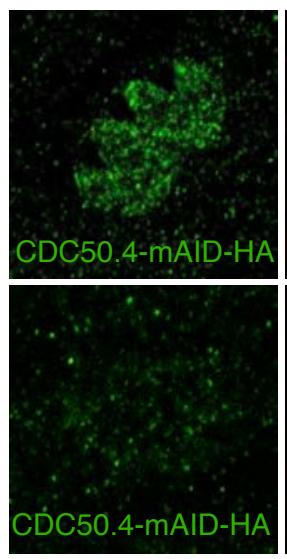

$G$

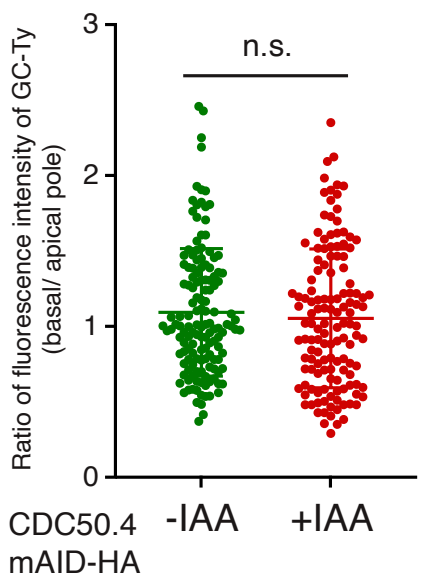

GC-TY

H CDC50.4-mAID-HA
B
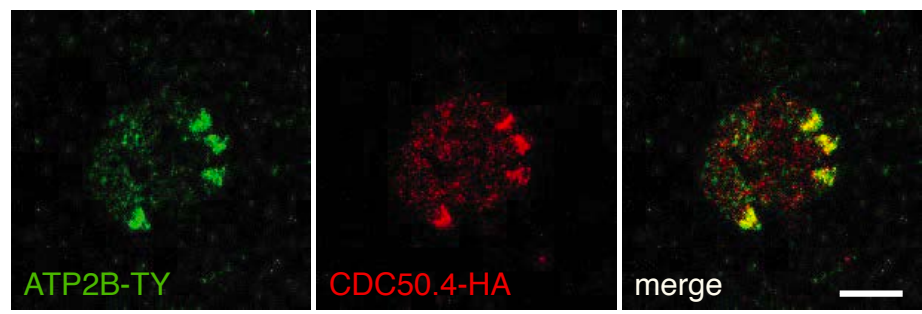

C

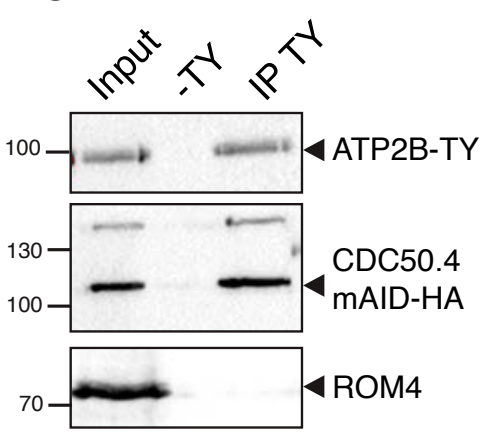

D

ATP2B-TY

CDC50.4-mAID-HA

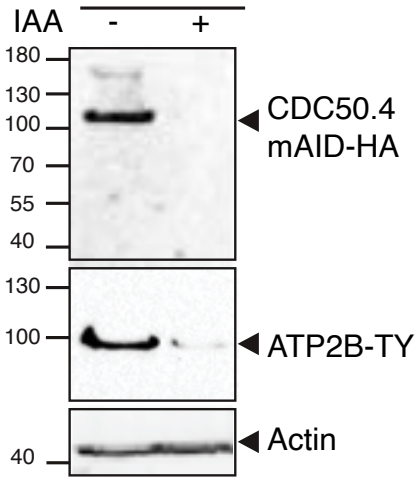

F
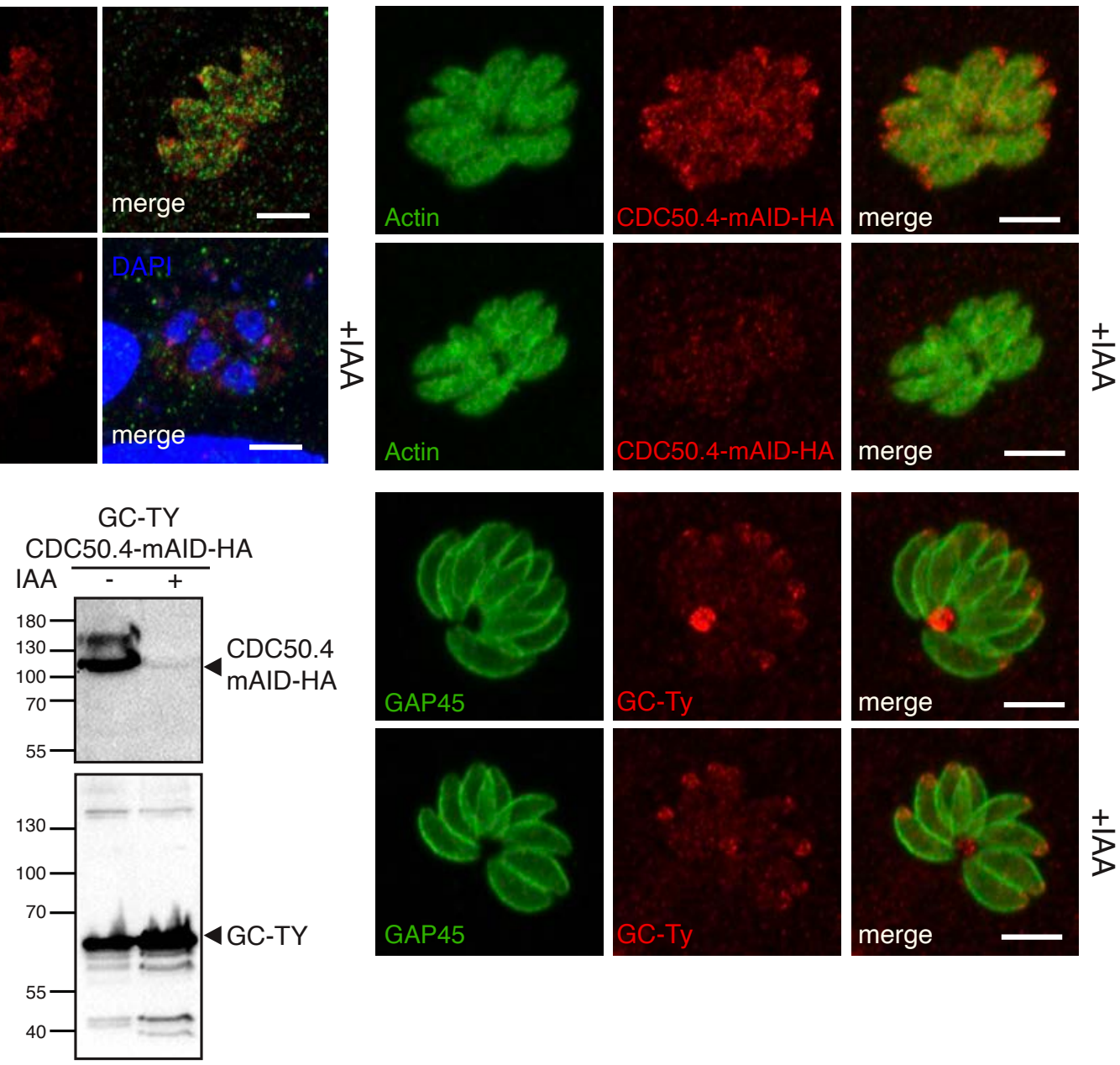
Figure 3

A

P ATP2B-mAID
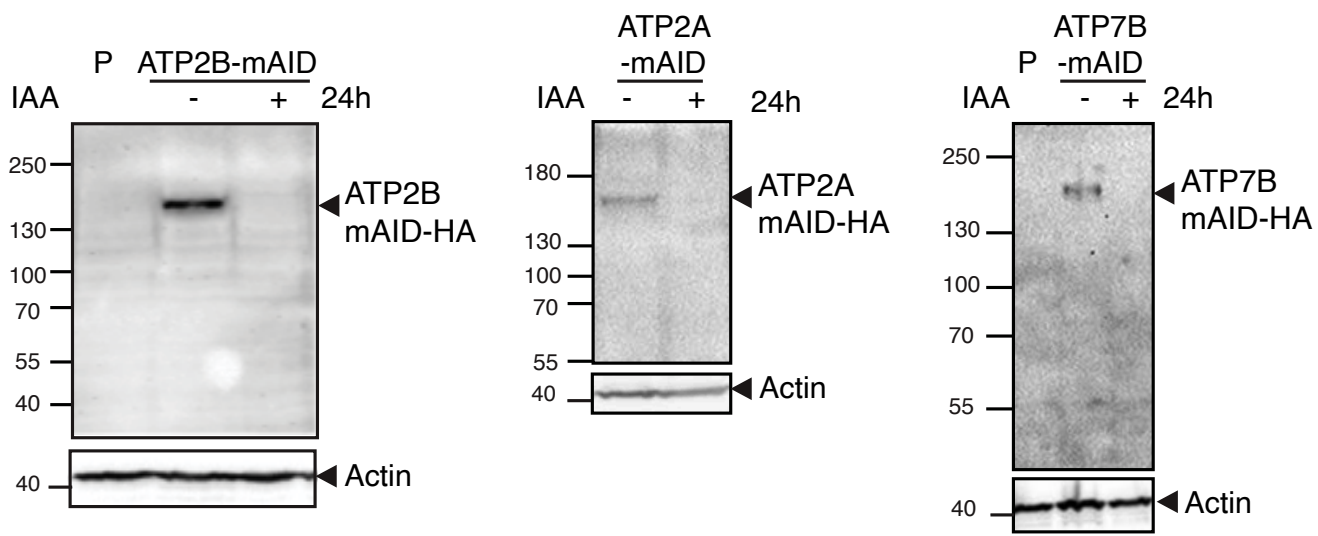

B
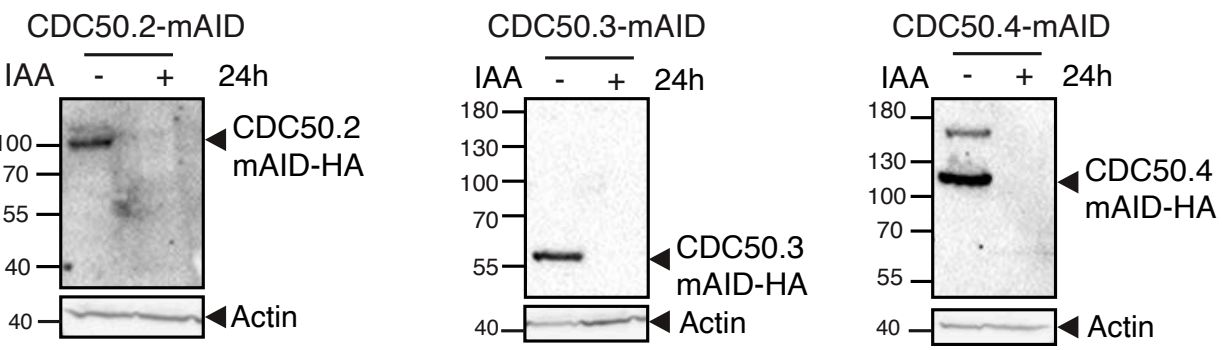

C

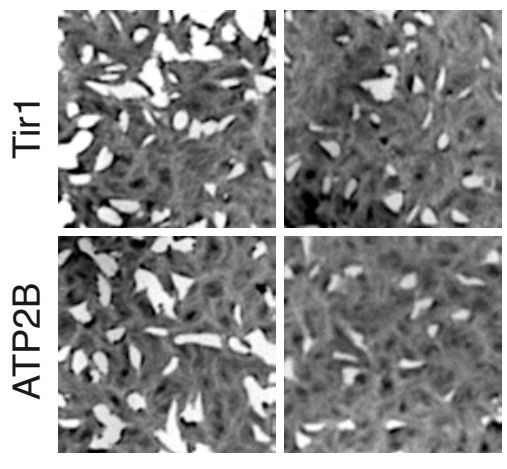

+ IAA

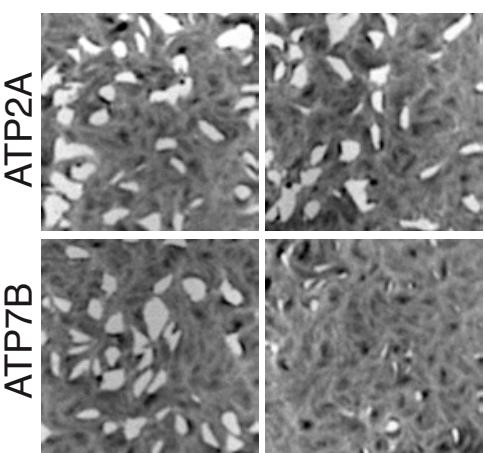

+ IAA

D
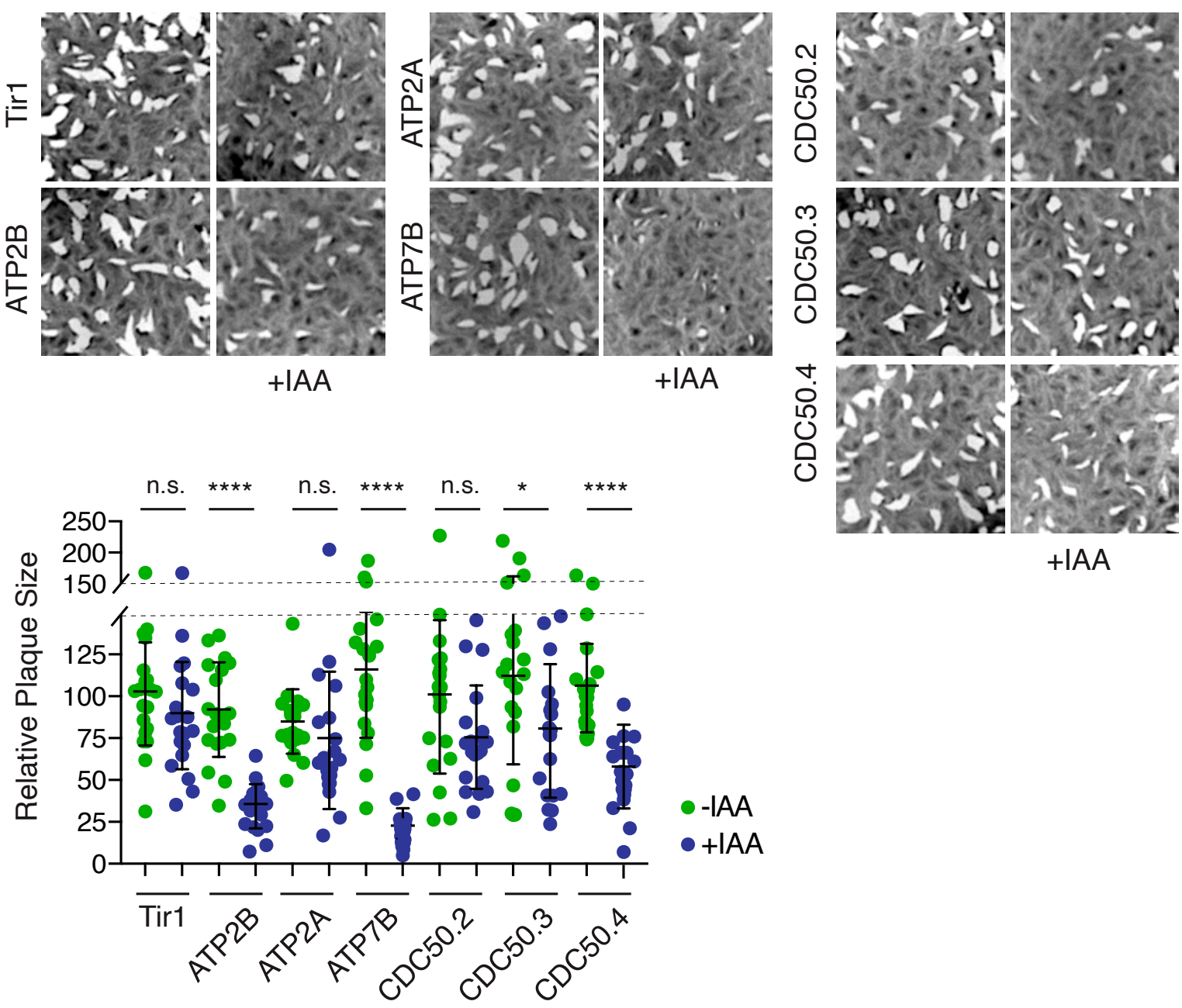
Figure 4
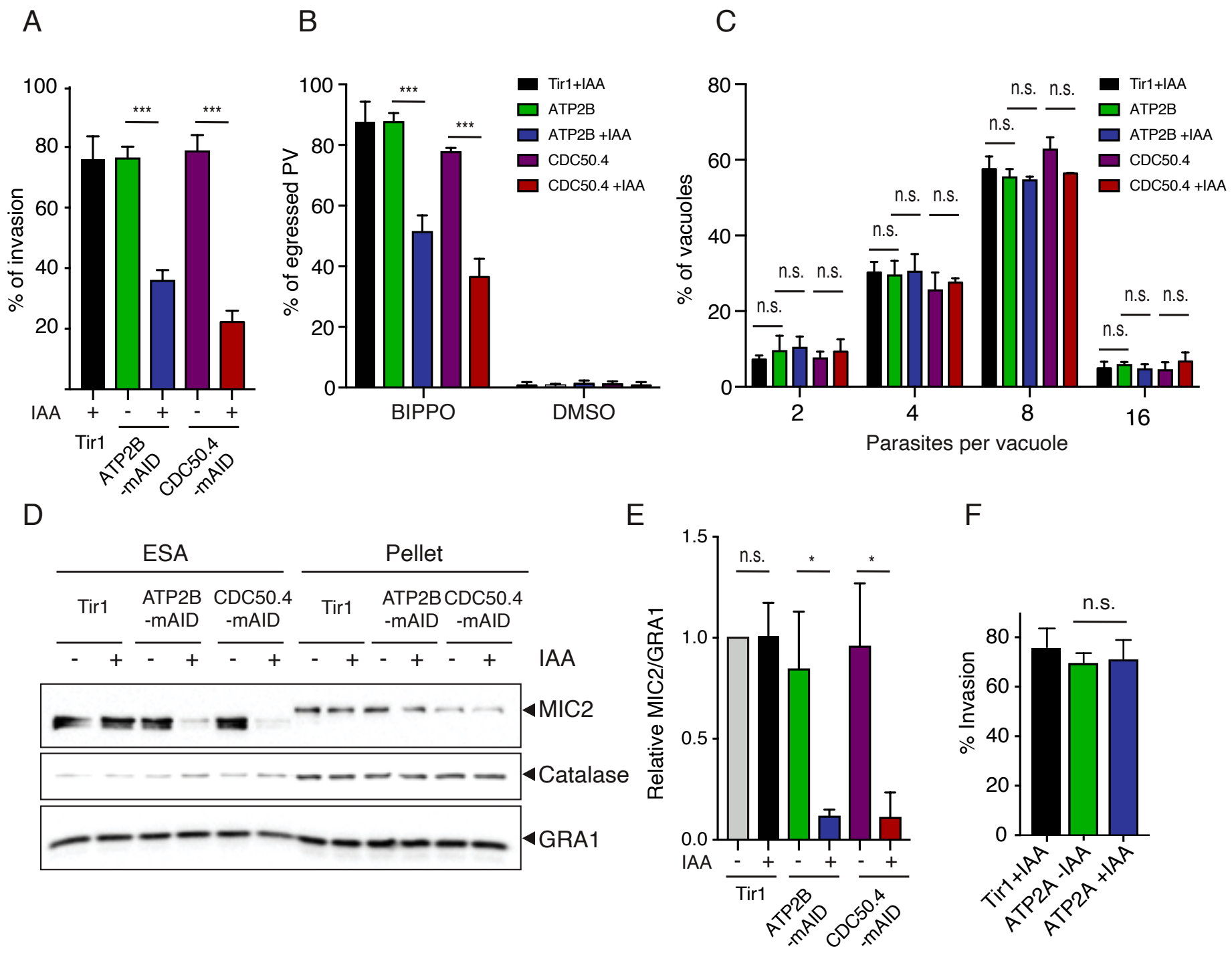

F

G

H
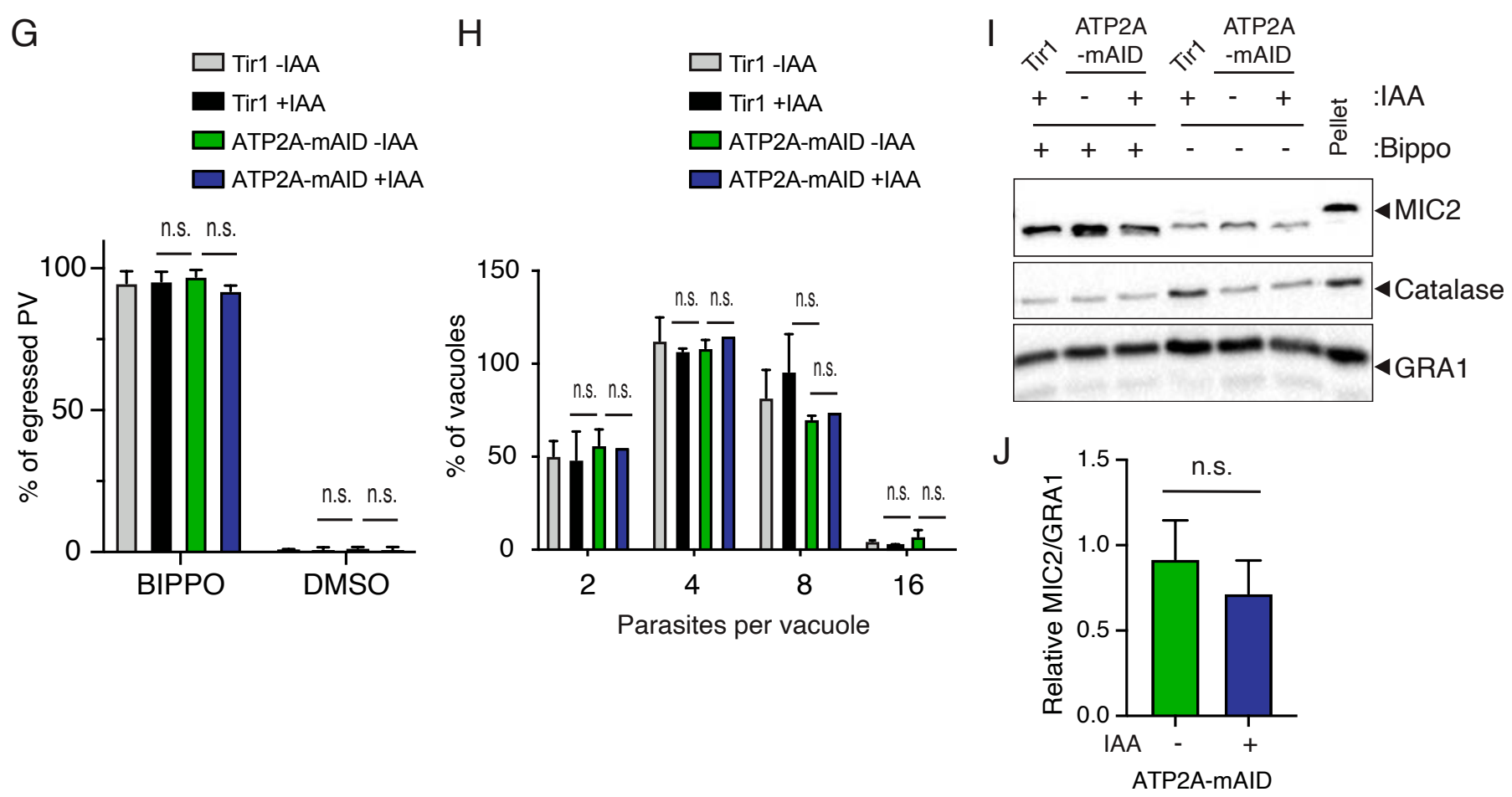
Figure 5
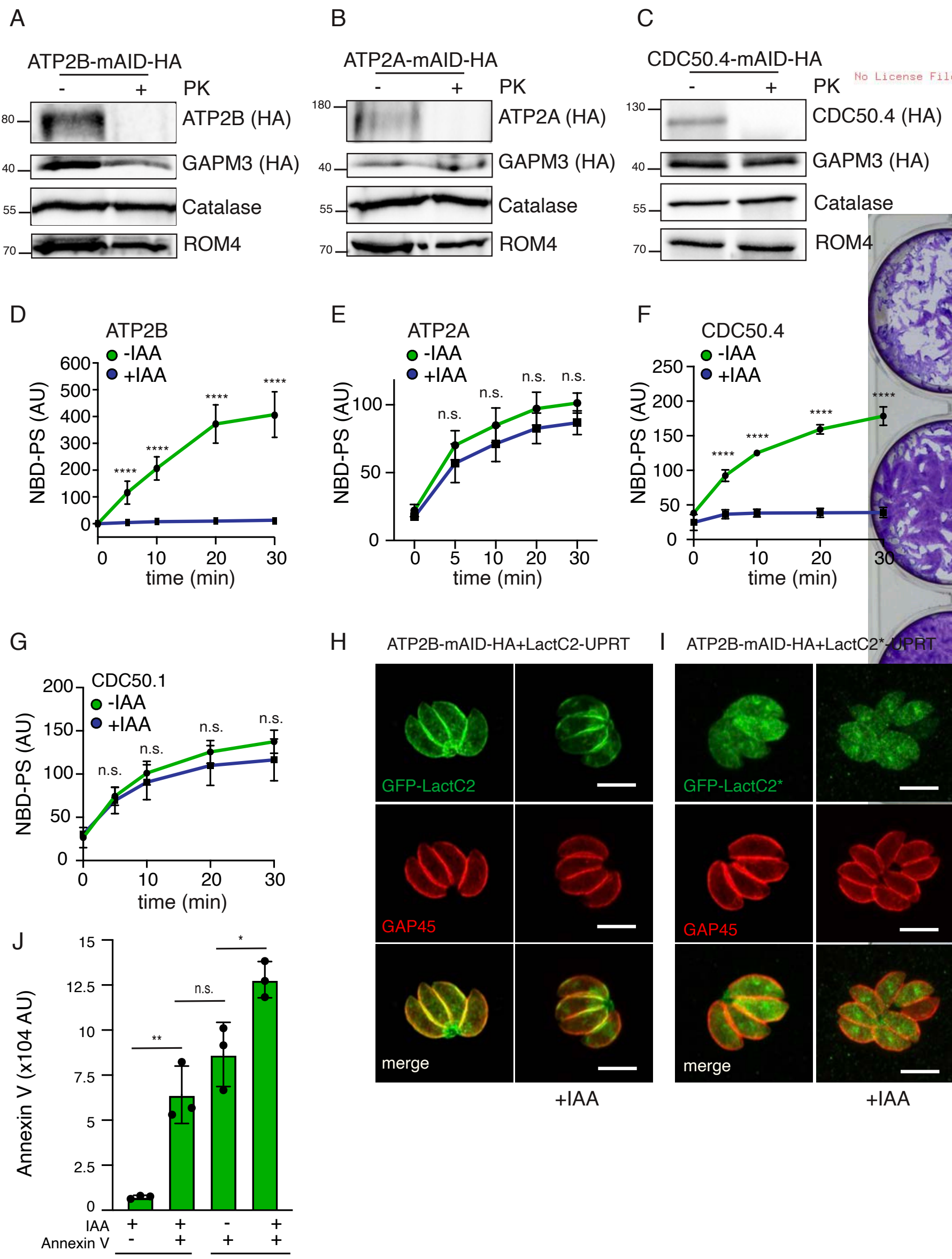

H ATP2B-mAID-HA+LactC2-UPRT I ATP2B-mAID-HA+LactC2*-UPRT
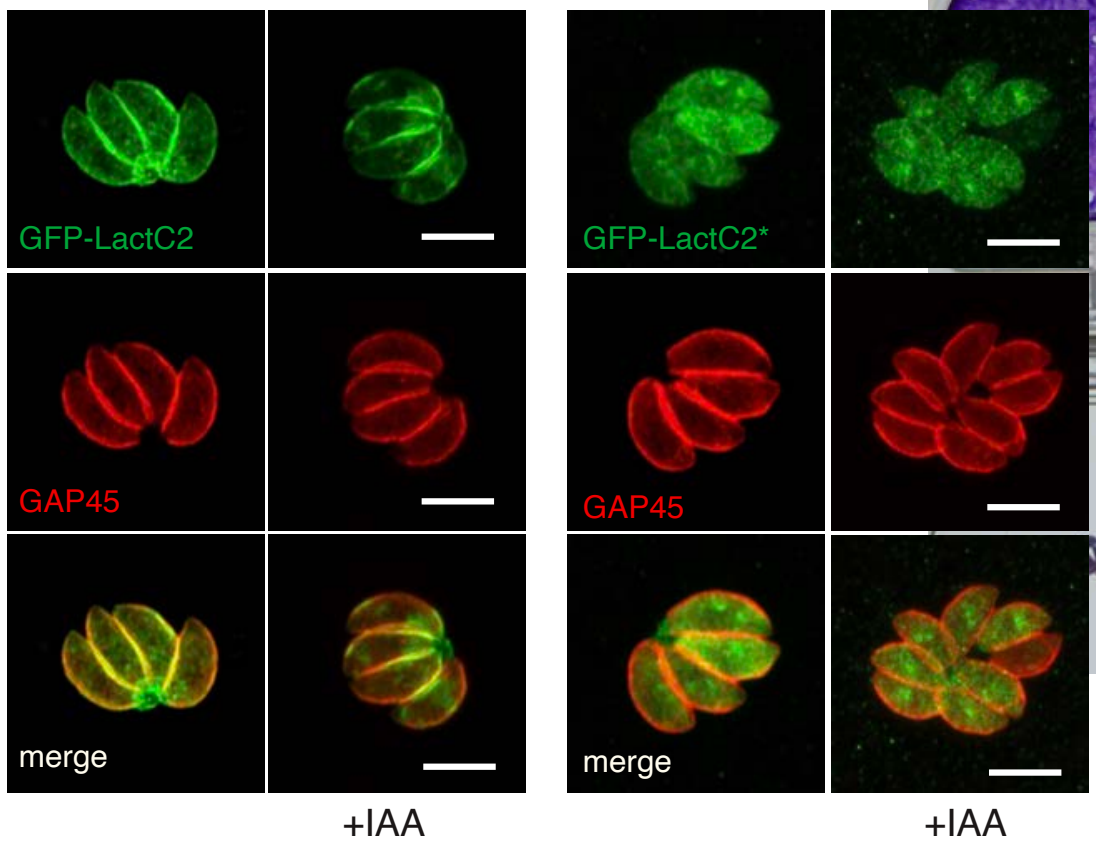

Parental ATP2B-mAID 
Figure 6

A

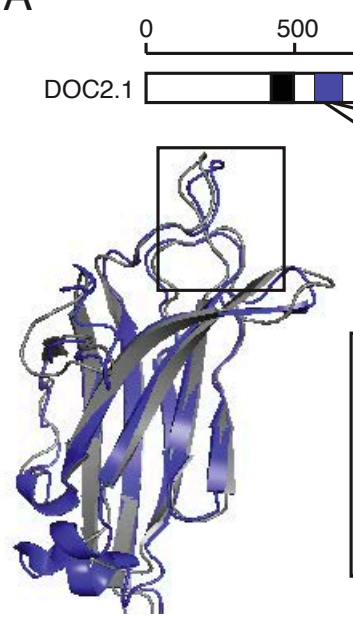

\begin{tabular}{l}
$500 \quad 1000 \quad 1500 \quad 2000$ \\
\hline
\end{tabular}
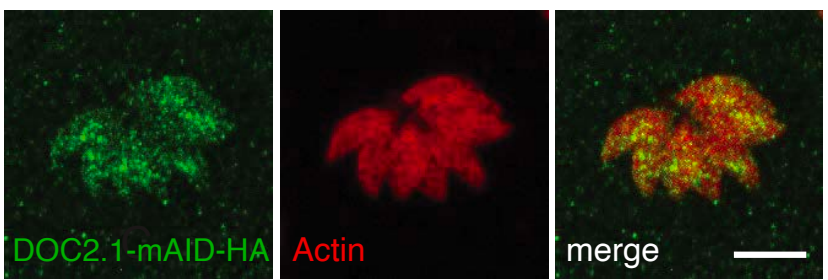

DOC2.1 QIVTPDNRPVVDSYVEV 595 SYT1 ELPALDMGGTSDPYVKV 184

PKC NLIPMDPNGLSDPYVKL 198

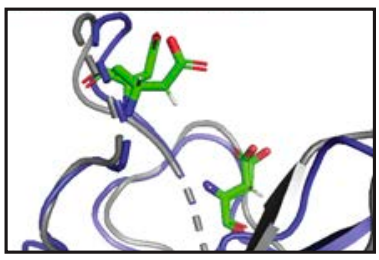

D

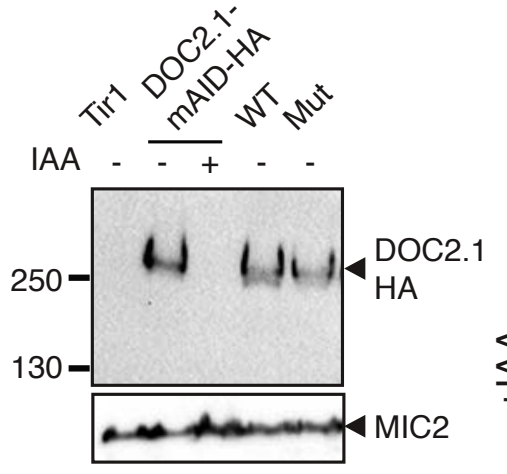

E

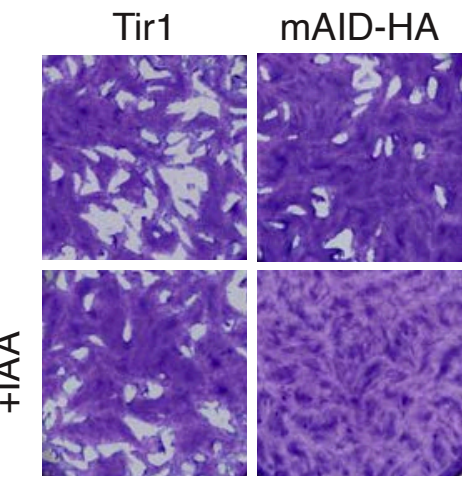

C Extracellular DOC2.1-mAID-HA
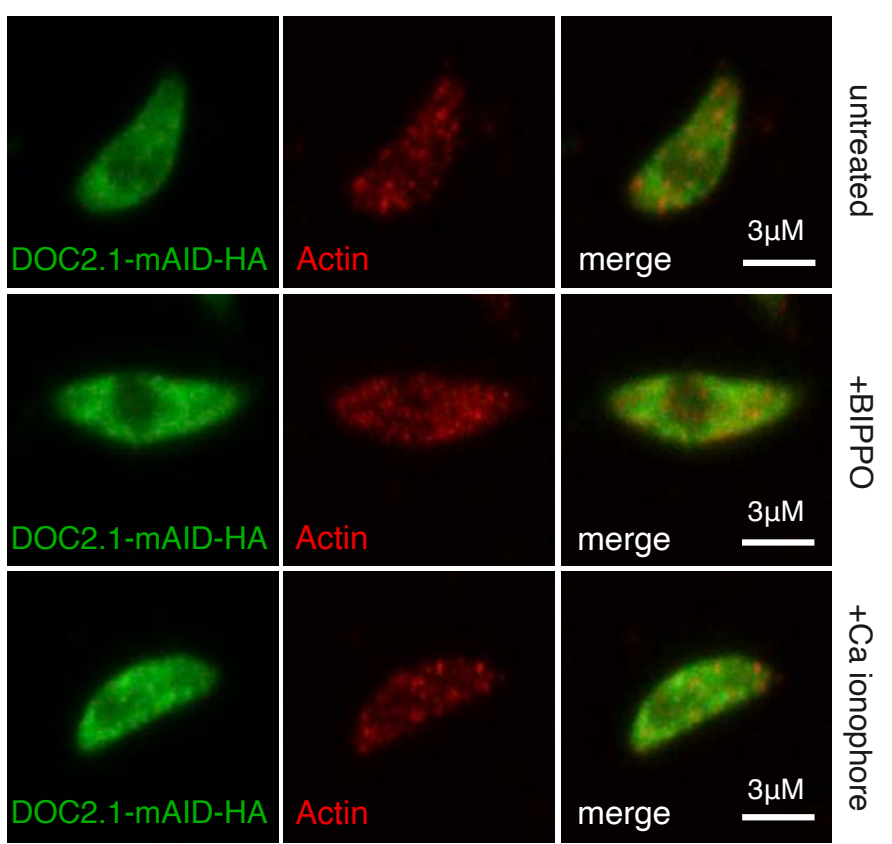

$\begin{array}{llll}F & \text { Doc2.1 Doc2.1 Doc2.1 }\end{array}$ (Parental) (WT)

(Mut)

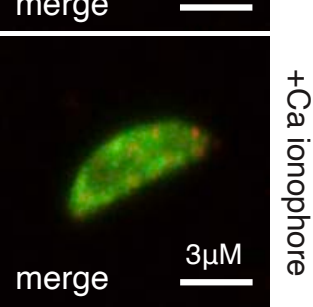

$\mathrm{H}$

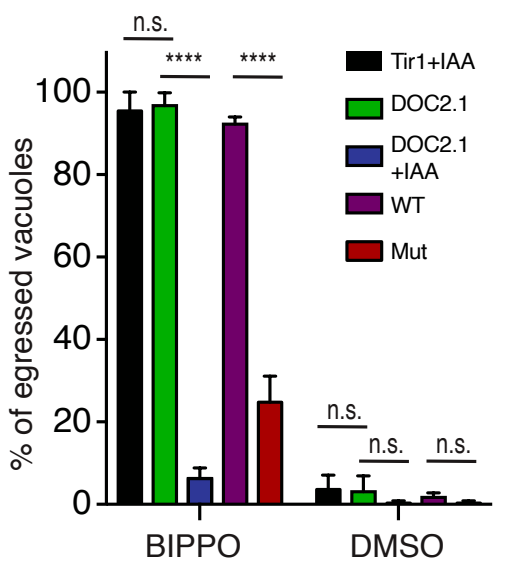

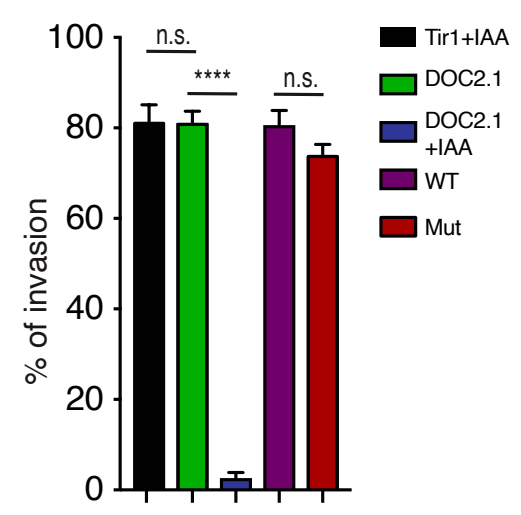

G

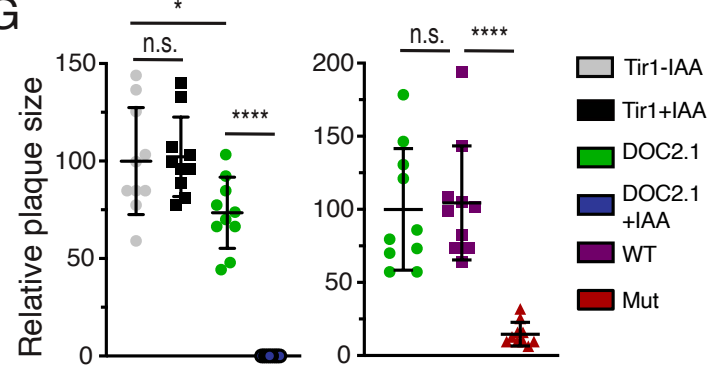

$J$

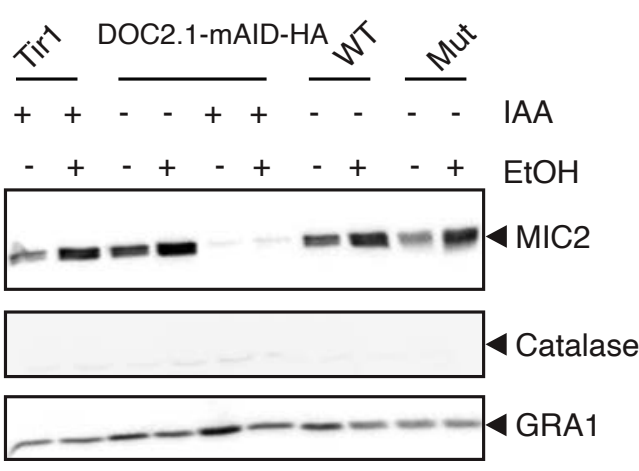

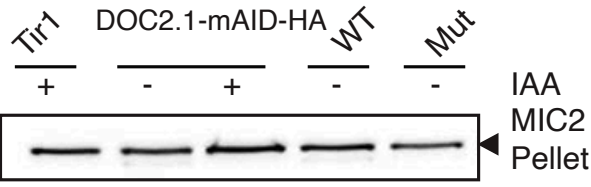

